\title{
Macrophages-Derived, LRG1-Enriched Extracellular Vesicles Exacerbate Aristolochic Acid Nephropathy Via A TGFBR1-Dependent Manner
}

\section{Wenjuan Jiang}

Anhui Medical University

Jiahui Dong

Anhui Medical University

Changlin Du

Anhui Medical University

Chuanting Xu

Anhui Medical University

Songbing Xu

Anhui Medical University

Rui Feng

Anhui Medical University

Cheng Huang

Anhui Medical University

Jun Li

Anhui Medical University

Taotao Ma ( $\square$ mataotao@ahmu.edu.cn )

Anhui Medical University https://orcid.org/0000-0003-2208-2505

\section{Research Article}

Keywords: Aristolochic acid nephropathy, NADPH oxidases 4, Macrophage, Tubular epithelial cells, Extracellular vesicles, Leucine-rich a-2-glycoprotein 1

Posted Date: June 30th, 2021

DOI: https://doi.org/10.21203/rs.3.rs-648122/v1

License: (1) (1) This work is licensed under a Creative Commons Attribution 4.0 International License.

Read Full License 


\section{Abstract}

Aristolochic acid nephropathy (AAN) is a progressive kidney disease caused by some herbal medicines, but treatment remains ineffective. We previously found NADPH oxidases 4 (NOX4), which regulates oxidative stress, play an important role in kidney injury model. However, its regulatory mechanism of action in AAN is still obscure. In this study, we established AAN model in vivo, a co-culture system of macrophage and TEC, and macrophage/TEC conditioned media culture model in vitro respectively. We found macrophages infiltration promoted injury $₫$ oxidative stress and apoptosis of TEC. Furthermore, the role of macrophage in AAN was dependent on macrophages-derived EV. Importantly, we found that macrophages-derived, Leucine-rich a-2-glycoprotein 1(LRG1)-enriched EV induced TEC injury and apoptosis of via a TGFßR1-dependent process. Mechanistically, macrophages-derived, LRG1-enriched EV mediating TECs injury by upregulating NOX4 in AAN model. This study may help design a better therapeutic strategy to treat AAN patients.

\section{Introduction}

Aristolochic acid (AA) is a generic term that describes a group of structurally related compounds found in Aristolochiaceae plants family.(Ma et al. 2021) 8-methoxy-6-nitro phenanthro-(3,4-d)-1,3-dioxolo-5carboxylic acid (AAI) is the major components of AA (Rosenquist et al. 2010). Aristolochic acid nephropathy (AAN) is a rapidly progressive tubulointerstitial disease induced by AA and effective treatment is lacking (Zhang et al. 2019, Debelle et al. 2008).

Several studies have shown that inflammation and oxidative stress are strongly linked to the pathogenesis of AA nephrotoxicity (Kim et al. 2019, Zeng et al. 2017). Macrophage infiltration is a prominent feature in both human and experimental AAN, and macrophage depletion studies have identified a pathologic role for macrophages in various animal models of renal inflammation and oxidative stress (Susnik et al. 2014, Honarpisheh et al. 2018). Pathologically, AAN is characterized by extensive tubular epithelial cells (TEC) injury in both patients and animal models of AAN (Wang et al. 2020a, Pozdzik et al. 2008). However, in AAN, it remains unclear how macrophage activation influences TEC injured and oxidative stress.

The cellular communication is an essential component in mammalian development and preservation of homeostasis (Gladka 2020). Beyond classical signaling through cell-cell contact and soluble factors, such as cytokines and inflammatory mediators, such intercellular communication also occurs through cellular release of extracellular vesicles (EV) (van Niel et al. 2018, Tkach and Thery 2016). EV is nanosized, membrane-bound vesicle released from different cell. Recent studies have revealed that EV may participate in renal tissue damage and regeneration through mediating inter-nephron communication (Karpman et al. 2017, Tang et al. 2019). Thus, the potential use of EV as therapeutic vector has gained considerable interest (Zhang and Liu 2021). 
NADPH oxidases 4 (NOX4), which regulates oxidative stress, play an important role in kidney injury model (Sundaresan et al. 2009). It is a novel therapeutic strategies targeting NOX4 in the treatment of certain types of renal diseases (Wang et al. 2020b, Ragot et al. 2016). However, its regulatory mechanism of action in AAN are still obscure.

In the current study, we found macrophages infiltration promoted injury and oxidative stress of TEC in AAN. Interestingly, AAl induced release of macrophages-derived EV. Macrophages-derived, LRG1-enriched EV promoting TECs injury is TGFBR1-dependent. Importantly, macrophages-derived, LRG1-enriched EV mediating TECs injury by upregulating NOX4 in AAN model.

\section{Materials And Methods}

\section{Experimental reagents}

Aristolochic acid I (Sigma-Aldrich, St. Louis, MO) was dissolved as previously described and used for cell

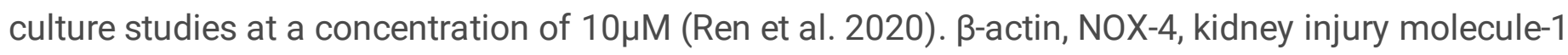
(KIM-1) primary antibodies and goat anti-rabbit or anti-mouse immunoglobulin $\mathrm{G}$ ( $\mathrm{lgG}$ ) horseradish peroxidase (HRP) secondary antibodies were purchased from Bioss (Beijing, China). TSG101, CD63, ARF6, Cleaved caspased-3, LRG1 antibody was obtained from Abcam (Cambridge, UK). Human recombinant LRG1 were purchased from Abcam (Cambridge, UK). Cr and BUN assay kit was purchased from Njjcbio (Nanjing, China). Annexin V-FITC/PI Apoptosis Detection Kit was purchased from Bestbio (Shanghai, China). Cytochalasin D was obtained from APExBIO (USA). PKH67 was obtained from Sigma (St. Louis, MO).

\subsection{Mice.}

C57BL/ 6 mice supplied by the Experimental Animal Center of Anhui Medical University were used to establish the AAN model. All the animal experiments were performed in accordance with the Regulations of the Experimental Animal Administration issued by the State Committee of Science and Technology of China. Efforts were made to minimize the number of animals used and their suffering. Animals were maintained in accordance with the guides of the Center for Developmental Biology, Anhui Medical University for the Care and Use of Laboratory. Animals and all experiments used protocols approved by the institutions' subcommittees on animal care. Mice were injected with AAI ( $5 \mathrm{mg} / \mathrm{kg}$, i.p.; Sigma-Aldrich, St. Louis, MO) every other day for 12 days. Kidney tissues and blood samples were obtained at 3, 9, 15, 21 and 27 days ( $n=6$ mouse per group). Control mice were treated with the same dosage of saline via intraperitoneal injection.

\subsection{Cell Lines and culture.}

HK-2 and mTEC, kindly provided by Prof. Huiyao Lan, were cultured in DME/F-12 (HyClone, Logan, UT, USA) supplemented with 10\% (vol/vol) heat-inactivated fetal bovine serum (Merck Millipore, Darmstadt, Germany) at $37^{\circ} \mathrm{C}$ in a humidified incubator under $5 \% \mathrm{CO}_{2}$. THP- 1 cells were cultured in RPMI- 1640 
(HyClone, Logan, UT, USA) supplemented with 10\% (vol/vol) heat-inactivated fetal bovine serum (Merck Millipore, Darmstadt, Germany) at $37^{\circ} \mathrm{C}$ in a humidified incubator under $5 \% \mathrm{CO}_{2}$. For all experiments, THP1 monocytes were differentiated into macrophages using phorbol 12-myristate 13-acetate $(5 \mathrm{ng} / \mathrm{mL})$.

\subsection{Primary cell isolation and culture.}

Bone marrow-derived macrophages (BMDM) were isolated from wild-type mice as previously described (Thery et al. 2006). Briefly, bone marrow was flushed from dissected mouse tibia and femur with sterile PBS. Cells were resuspended and plated in RPMI-1640 (Gibco, Carlsbad, CA) supplemented with $10 \%$ heat-inactivated fetal bovine serum (FBS), $100 \mathrm{U} / \mathrm{mL}$ penicillin, $100 \mu \mathrm{g} / \mathrm{mL}$ streptomycin, and M-CSF (10 $\mathrm{ng} / \mathrm{mL}$ ) conditioned media. BMDM were used for experiments after a differentiation period of 7 days. For real-time quantitative PCR analysis, $3 \times 10^{5}$ cells were plated per well into 12-well plates. The next day, BMDM were treated with EV in RPMI 1640 medium for 8h before RNA isolation.

Extracellular vesicle isolation. Renal primary epithelial cell and macrophage derived EV isolated from T2D mice were collected over a $24 \mathrm{~h}$ time period in EV-depleted complete media, which was prepared by overnight centrifugation at $100,000 \times \mathrm{g}$ at $4^{\circ} \mathrm{C}$. Unless stated otherwise, $\mathrm{EV}$ was isolated from cell culture medium by differential ultracentrifugation using a modified version of a protocol by Thery et al (Thery, Amigorena 2006). Collected medium was depleted of cells and cell debris by consecutive, low-speed centrifugations $(2,000 \times \mathrm{g}$ for $15 \mathrm{~min}$ and $16,000 \times \mathrm{g}$ for $20 \mathrm{~min})$. The supernatants obtained were carefully collected and centrifuged for $90 \mathrm{~min}$ at $100,000 \times \mathrm{g}$ at $4^{\circ} \mathrm{C}$. Pellets from this centrifugation step were washed in PBS, pooled, and centrifuged again for $90 \mathrm{~min}$ at $100,000 \times \mathrm{g}$ at $4^{\circ} \mathrm{C}$. The obtained pellets were resuspended in lysis buffer (see below), PBS solution or RPMI-1640 medium, depending on subsequent experiments. EV solutions intended for cell treatment were sterile filtered through $0.22 \mu \mathrm{m}$ syringe filter. Resuspended EV were either used for subsequent analysis or aliquoted and stored at $-80^{\circ} \mathrm{C}$. For isolation of EV derived from primary cells, a commercially available kit from Invitrogen (Carlsbad, CA) was utilized.

\subsection{Electron microscopy.}

Isolated EV were fixed in $2 \%$ paraformaldehyde in $0.1 \mathrm{M}$ phosphate buffer overnight at $4^{\circ} \mathrm{C}$. The samples were then placed on Formvar-carbon-coated grid and air dried for $20 \mathrm{~min}$. After being rinsed with PBS, grids were transferred to $1 \%$ glutaraldehyde for $5 \mathrm{~min}$ and washed with distilled water. The grids were first contrasted with uranyl-oxalate solution and then contrasted and embedded in a mixture of $4 \%$ uranylacetate and $2 \%$ methylcellulose (1:9 ratio). The grids were air dried and visualized with a JEOL 1400 electron microscope (JEOL USA, Peabody, MA) at 80 kV. For immunogold staining, grids were block with $10 \%$ FBS for 20 min followed by overnight incubation at $4^{\circ} \mathrm{C}$ with primary anti-TRAIL and anti-LRG1 antibody diluted 1:20 in blocking solution overnight. Next, grids were incubated with secondary antibody for $1 \mathrm{~h}$. In negative control samples, primary antibody was omitted. Samples were then labeled with protein A-10-nm gold for $1 \mathrm{~h}$. The grids were contrasted and embedded with a mixture of $4 \%$ uranyl acetate and $2 \%$ methylcellulose (1:9 ratio) and observed as described above. 
4.6 Nanoparticle tracking analysis.

Concentration and size distribution of isolated EV were assessed by nanoparticle tracking analysis (NTA) using NanoSight NS300 instrumentation (NanoSight, Amesbury, UK). EV samples were diluted with PBS at a range of concentrations between $4 \times 10^{8}$ and $8 \times 10^{8}$ particles per milliliter in a total volume of 1 milliliter. Each sample was continuously run through a flow-cell top-plate set up to $23.3^{\circ} \mathrm{C}$ using a syringe pump at a rate of $25 \mu \mathrm{L} / \mathrm{min}$. At least three videos of 30 seconds documenting Brownian motion of nanoparticles were recorded and at least 1000 of completed tracks were analyzed by NanoSight software (NTA 2.3.5).

\subsection{Cell EV uptake.}

THP-1-derived purified EV were labeled with a green fluorescent dye PKH67 (Sigma) and washed by a $100,000 \times \mathrm{g}$ spin. Differentiated HK-2 cells were then incubated with these $\mathrm{EV}\left(10^{10} / \mathrm{mL}\right)$ for $1 \mathrm{~h}$ (with or without $10 \mu$ Mcytochalasin D pre-Hirsova et al. treatment) and their cellular internalization was observed using an LSM880 confocal microscope (Carl Zeiss, Jena, Germany).

\subsection{Flow Cytometry.}

The extent of the programmed cell death was detected by flow cytometry (CytoFlex, Beckman Coulter, USA) using AV-FITC/PI apoptosis detection kit (Bestbio, Shanghai, China). To evaluate the apoptosis level of HK-2, the attached and supernatant cells were stained with $5 \mu$ l of Annexin V-FITC and10 $\mu \mathrm{PI}$ in the dark, detected by flow cytometry, and analyzed using CytExpert 2.1 software (CytExpert, Beckman Coulter, USA).

\subsection{Transient transfection.}

A small interfering RNA (siRNA) was used to silence TGFBR1 in THP-1 cells. Cells transfected with scramble siRNA were used as control. Cells were grown in 60-mm dishes and transiently transfected with Smart Pool siRNA (5 nM, Hanbio) using LipoFiter 3.0 (Hanbio). Experiments were performed $48 \mathrm{~h}$ after transfection.

\subsection{Immunofluorescence Staining.}

Sections were blocked with $10 \%$ bovine serum albumin (BSA) solution to avoid non-specific staining. Sections were incubated with rabbit polyclonal primary antibodies against KIM-1 (1:500). Sections were incubated overnight at $4^{\circ} \mathrm{C}$, followed by incubation with anti-rabbit Cy-3 (1:200)-conjugated secondary antibodies and nuclei staining with 4',6-diamidino-2-phenylindole (DAPl; Beyotime Biotechnology, Shanghai, China). Stained sections were examined with an inverted fluorescence microscope (Carl Zeiss Axio Vert.A1, Jena, Germany).

\subsection{Histopathology.}


Renal tissues of mice were fixed in $4 \%$ paraformaldehyde for $24 \mathrm{~h}$ immediately following killing, processed for histological examination according to a conventional method, and stained with PAS, H\&E and F4/80. The slides were scored in a blinded manner and de-identified.

\subsection{TUNEL Assay.}

Renal cell apoptosis was examined by TUNEL assay using the One step TUNEL Apoptosis Assay Kit from Beyotime Biotechnology (Beyotime, Jiangsu, China). Briefly, cells were fixed with $4 \%$ paraformaldehyde in PBS and then exposed to the TUNEL reaction mixture containing TM red-labeled dUTP. Finally, samples were counterstained with 4',6-diamidino-2-phenylindole (DAPI). TUNEL-positive nuclei were identified by fluorescence microscopy.

\subsection{Western Blot.}

Whole extracts were separated by 10 or $12 \%$ sodium dodecyl sulfate polyacrylamide gel electrophoresis (SDS-PAGE), transferred to a polyvinylidenedifluoride membrane, which were incubated with primary antibodies against LRG1/ NOX-4/ KIM-1/ TSG101/ ARF6/ CD63/ cleaved-caspased-3/ $\beta$-actin (1:500; Santa Cruz biotechnology, Dallas, TX, USA). The membranes were then washed in TBS-Tween 20 and incubated with secondary antibodies correspondingly. After extensive washing in TBS-Tween 20, protein bands were visualized with ECL chemiluminescent kit (ECL-plus; Thermo Fisher Scientific, Pittsburgh, PA, USA).

\subsection{Real-Time Reverse Transcriptase-PCR.}

Total RNA was collected from kidney tissues, THP-1 and BMDM cells using TRIzol reagents (Invitrogen). First-strand cDNA was synthesized using Thermoscript RT-PCR synthesis kit (Fermentas, Pittsburgh, PA, USA) according to the manufacturer's instructions. Real-time quantitative PCR analyses for mRNA were performed using Thermoscript RT-qPCR kits in an ABI Prizm step-one plus real-time PCR System (Applied Biosystems, Foster City, CA, USA). The products were used as templates for amplification using the SYBR Green PCR amplification reagent (Qiagen, Valencia, CA, USA) and gene-specific primers. Relative expression levels were calculated according to the standard $2^{-\Delta \Delta C t}$ method (Schmittgen and Livak 2008). The forward and reverse primers used for PCR were as listed in Supporting Information Tab S1.

\subsection{Statistical Analysis.}

Data are expressed as the means \pm SEM and represent at least three independent experiments.

Differences between two groups were compared using the two-tailed Student's t-test. Differences between multiple groups were compared using one-way analysis of variance followed by Student's t-test. Differences were considered significant at $\mathrm{P}<0.05$. All analyses were performed using GraphPad Prism 5.0 software (San Diego, CA).

\section{Results}




\subsection{Aristolochic acid induces kidney injury in a time-dependent manner.}

To determine renal response to aristolochic acid treatment, kidney tissues were harvested at 3, 9, 15, 21 and 27days after aristolochic acid (AA) administration (Fig. 1A). Administration of aristolochic acid to C57BL/6 mice resulted in typical features of aristolochic acid nephropathy (AAN). As shown in Fig. 1B, tubular damages with atrophy, dilatation and bared tubular basement membrane were raised along with the increased treatment time. Consistent with the severity of tubular damage seen on PAS stained sections, KIM-1, an established biomarker for renal proximal tubule injury, was barely detectable in the kidney of vehicle mice but dramatically increased after AAl administration in a time-dependent. In addition, intervention with AAI treatment substantially increased KIM-1 expression seen over days 15 to 27 (Fig. 1C). Renal tubular epithelial cell (TEC) apoptosis is the common histopathological feature of AAI nephrotoxicity. A significant increase in cleavedcaspase-3 was evident on day 21(Fig. 1C). Then, we also evaluated the renal cell turnover by staining $\mathrm{TUNEL}^{+}$cells in mice kidneys. Similar result was found that the levels of apoptosis were increased in AAN mice along with the increased AAl treatment time (Fig. 1D). These data indicated that aristolochic acid induced kidney injury in a time-dependent manner.

\subsection{Macrophage infiltration promotes AAl-induced injury.}

Macrophage infiltration is a hallmark of AAN and is believed to promote TEC damage in AAN. A prominent interstitial accumulation of $\mathrm{F} 4 / 80^{+}$macrophages was seen in AAl treated mice (Fig. 2A). These infiltrates were accompanied by up-regulation of the chemokine (C-X-C motif) ligand 12 (CXCL12) and pro-inflammatory Interleukin $1 \beta$ (IL-1 $\beta$ ) at the mRNA level (Fig. 2B and 2C).

To explore the effects of macrophage on tubular epithelial cells (TEC), we firstly employed a co-culture system as shown schematically in Fig. 2D. The human monocytic cell line (THP-1), which can be differentiated into macrophage-like cells,in the lower chamber of a trans-well plate were co-cultured with TEC in the upper chamber. The co-culture system was treated with AAI $(40 \mu \mathrm{M})$ for 24 hours and harvested for analysis. As shown in Fig. 2E, treated with AAI induced significantly up-regulated levels of CXCL12 and IL-1 $\beta$ in the co-cultured macrophages. Additionally, treatment with AAI up-regulated the protein level of KIM-1 in the co-culture TECs (Fig. 2F). Moreover, western blot analysis indicated that the level of cleaved caspase-3 was markedly increased in the co-culture TECs (Fig. 2F). The same change was also found in the level of apoptosis in the co-culture TEC by flow cytometric analysis (Fig. 2G).

Secondly, THP-1/HK-2 conditioned media culture model was also established (Fig. 3A). As shown in Fig. 3B, AAl significantly increased the CXCL12 and IL-1 $\beta$ expression of THP-1 cells. Interestingly,a dose of AAI $(40 \mu \mathrm{M}, 24 \mathrm{~h})$ did not induce HK-2 injury, but the level of KIM-1 and cleaved caspase-3 were increased with addition of AAI-CM-induced group (conditioned medium from AAI-induced THP-1 cells added to AAl-induced HK-2 cells) compared to AAl-induced groups (Fig. 3C). Furthermore, immunofluorescence staining showed the level of cleaved caspase-3 was largely up-regulated in AAI-CMinduced group (Fig. 3D). Flow cytometric analysis also identified a significant increase of cell apoptosis in AAI-CM-induced group compared to other groups (Fig. 3E). 
Furthermore, BMDM/mTEC conditioned media culture model was also established. Similar results were demonstrated CM (conditioned medium from AAl-induced BMDM cells added to AAl-induced mTEC cells) treatment promoted injury and apoptosis of TEC induced by AAI (Suppplementqal Fig. 1A and 1B).

These results above indicated conditioned medium from AAl-induced macrophage promoted the role of AAl in renal tubular epithelial cell injury.

\subsection{Macrophages-derived EV promote AAl-induced injury.}

Cell-to-cell communication via direct contact or through soluble factors is of vital importance for multicellular organisms. Interestingly, upon AAI treatment, released EV were isolated from THP-1 cell culture media and quantified via nanoparticle tracking analysis (NTA). Over a 24-hour incubation period, AAl induced an approximately 2.5-fold increase in release of EV in THP-1 cells (Fig. 4A). The size characteristics of the released EV were confirmed by electron microscopy (Fig. 4B). Immunoblot analysis indicated that isolated EV contained established exosomal and microvesicular markers such as tumor susceptibility gene 101 (TSG101), adenosine diphosphateribosyltion factor 6 (ARF6), CD63 and $\beta$-actin (Fig. 4C). Consistent with THP-1 cell data, AAI had the similar effect on EV release in BMDM cells (Fig. 4D and $4 \mathrm{E})$.

Secondly, the data above provided evidence suggesting AAI induced release of macrophages-derived EV, which prompted us to investigate whether this is due to EV transfer. Purified EV were isolated from primary macrophages of AAI -induced, 27-day AAN model mice kidney tissues and fluorescence-labelled the EV with PHK67. Then PHK67-labelled EV were injected into AAl-induced, 3 -day mice, which could not establish AAN model, via renal in situ injection (Fig. 4F). It was found that fluorescence-labelled macrophage-derived EV were localized in kidney (Fig. 4G). Most importantly, AAl-induced, macrophagederived EV enhanced the levels of KIM-1 and cleaved caspase-3 in AAl-induced, 3 -day mice (Fig. $4 \mathrm{H}$ ). Furthermore, TUNEL staining also revealed that AAl-induced, macrophage-derived EV significantly promoted apoptosis in the kidney tissues of AAl-induced, 3 -day mice (Fig. 4I).

Thirdly, AAl-induced HK-2 was incubated with AAI-treated, THP-1-derived EV (Fig. 5A). The levels of KIM-1 and cleaved caspase-3 expression in AAl-induced HK-2 were no significantly increased, while these levels were sharply increased in AAl-induced HK-2 cells with addition of AAl-treated, THP-1-derived EV $\left(\mathrm{EV}_{\mathrm{a}}\right)$, as confirmed in Fig. 5B. Similar analysis of the level of cleaved caspase-3 using immunofluorescence staining was shown in Fig. 5C. In addition, AAl-induced HK-2 cells with addition of AAl-treated, THP-1derived $E V\left(E V_{a}\right)$ promoted much higher apoptosis level in Fig. 5D.

To further test hypothesis, we incubated AAI-induced mTEC with purified EV released from AAl-treated BMDM $\left(10^{10} / \mathrm{ml}\right)$ (Suppplementqal Fig. $\left.2 \mathrm{~A}\right)$. Similar results were also shown by immunoblot and immunofluorescence staining in Suppplemental Fig. 2B and 2C.

Last but not the least, to demonstrate that EV-unrelated fraction was not responsible for TEC injury, we destroyed EV by boiling. It was observed that this treatment abolished their stimulatory activity, as 
confirmed in Fig. 6A. We then examined whether phagocytosis was required for TEC injury by EV. It was demonstrated that the level of cleaved Caspase-3 was no change with addition of Cytochalasin D. Cytochalasin D, a pharmacologic inhibitor of phagocytosis, efficiently inhibited phagocytosis of fluorescently labeled EV but had no effect on AAI-treated, THP-1-derived EV (EV $\mathrm{a}_{\mathrm{a}}$ ) induced injury (Fig. 6B and $6 \mathrm{C}$ ). These data suggest that macrophages-derived EV promotes AAl-induced injury and EV-TEC interaction at the level of the plasma membrane is likely responsible for TEC injury.

\subsection{Macrophages-derived, LRG1-enriched EV promoting TECs injury is TGFBR1-dependent.}

Our previous study revealed that LRG1 was highly elevated in CP-induced renal damage(Jiang et al. 2020a). Interestingly, we isolated primary renal macrophage derived EV from AAN model and found the level of LRG1 was significantly increased by western blot analysis (Fig. 7A). The same changes were also found in immunogold labeling and electron microscopy in Fig. 7B.

Consistent with in vivo data, LRG1 was up-regulated in AAl-induced THP-1 and BMDM by real-time PCR analysis (Fig. 7C and 7D). Furthermore, LRG1 was also up-regulated in AAl-induced THP-1-derived EV and AAl-induced BMDM-derived EV by western blot analysis (Fig. 7E).

To explore the role of LRG1 on TEC injury, we treated HK-2 cells with recombinant human LRG1. LRG1pretreated, AAI-induced HK-2 cells expressed much higher levels of KIM-1 than AAI treated group, whereas AAl treatment alone in the absence of LRG1 did not affect the basal level of KIM-1 by western blot (Fig. 7F).

LRG1 could bind directly to the TGF $\beta$ receptor 1(TGF $\beta R 1$ ), however it is unknown whether LRG1-enriched EV could promote TEC injury via TGF $\beta R 1$. Next, we treated HK-2 cells with siRNA-TGF $\beta R 1$ for 24 hours (Fig. 7G). As illustrated in Fig. 7H, siRNA-TGFßR1 suppressed the expression of KIM-1 in TGF $\beta 1^{-/}{ }^{-H K}-2$ cells.

These data suggest that macrophages-derived, LRG1-enriched EV promoting TEC injury is TGF $\beta R 1$ dependent.

\subsection{Macrophages-derived, LRG1-enriched EV mediate TEC injury by upregulating NOX4.}

Administration of aristolochic acid to C57BL/6 mice resulted in typical features of oxidative stress in AAN. GSH and SOD levels were markedly down-regulated in AAl-induced group, while MDA levels were significantly up-regulated in a time-dependent manner (Fig. 8A-8C). Furthermore, intervention with AAI treatment substantially increased NOX4, a major source of reactive oxygen species (ROS) in the kidney, seen over days 9 to 27 (Fig. 8D). To explore the effects of macrophage on oxidative stress, we employed the co-culture system above. Treatment with AAI up-regulated the protein level of NOX4 in the co-culture HK-2 (Fig. 8E). Moreover, THP-1/HK-2 conditioned media culture model was also established to get the similar result of NOX4 (Fig. 8F). Similar results were also shown in BMDM/mTEC conditioned media culture model (Supplemental Fig. 3A.). 
It was found that macrophages-derived EV promoted AAl-induced injury. Purified EV, isolated from primary macrophages of AAI -induced, 27-day AAN model mice kidney tissues, were injected into AAIinduced, 3-day mice via renal in situ injection. Interestingly, AAl-induced, macrophage-derived EV enhanced the levels of NOX4 in AAl-induced, 3-day mice (Fig. 8G). Consistent with in vivo data, the level of NOX4 expression in AAl-induced HK-2 was no significantly increased, while these levels were sharply increased in AAl-induced HK-2 cells with addition of AAl-treated, THP-1-derived EV (EV $\mathrm{a}_{\mathrm{a}}$, as confirmed in Fig. 8H. Similar results were also shown in Supplemental Fig. 3B.

To demonstrate that an EV-unrelated fraction was not responsible for oxidative stress, we destroyed EV by boiling. It was observed that this treatment abolished their stimulatory activity, as confirmed in Fig. 9A. We then examined whether phagocytosis was required for oxidative stress by EV. It was demonstrated that the level of NOX4 was no change with addition of Cytochalasin D. Cytochalasin D had no effect on AAl-treated, THP-1-derived EV (EV $\left.{ }_{a}\right)$ induced oxidative stress (Fig. 9B).

To explore the role of LRG1 on oxidative stress, we treated HK-2 cells with recombinant human LRG1. LRG1-pretreated, AAl-induced HK-2 cells expressed much higher levels of NOX4 than AAl treated group, whereas AAl treatment alone in the absence of LRG1 did not affect the basal level of NOX4 by western blot and immunofluorescence staining (Fig. 9C and 9D). In addtion, as illustrated in Fig. 9E and 9F, siRNATGF $\beta R 1$ suppressed the expression of NOX4 in TGF $\beta R 1^{-/-} \mathrm{HK}-2$ cells.

These data suggest that LRG1-enriched EV from macrophages promote oxidative stress via a TGFBR1dependent manner in AAN model.

\section{Discussion}

In the current study, we found aristolochic acid induced kidney injury in a time-dependent manner. Macrophages infiltration promoted kidney injury,oxidative stress and apoptosis of TEC. Furthermore, the role of macrophage in AAN was dependent on macrophages-derived EV. Importantly, we found that macrophages-derived, LRG1-enriched EV induced TEC injury via a TGF $\beta R 1$-dependent process by upregulating NOX4 in AAN model.

AAl-induced oxidative stress serves an important role in the development of renal injury (Jadot et al. 2017, Wu et al. 2015). Various studies have demonstrated that oxidative stress can induce cell injury and apoptosis(Yaribeygi et al. 2018). NADPH oxidases are a major source of reactive oxygen species (ROS) in the kidney in normal and pathological conditions (Sedeek et al. 2013). Among NADPH oxidase isoforms, NADPH oxidase4 (NOX4), which regulates oxidative stress, is highly expressed in the kidney and has an important role in kidney diseases (Yoo et al. 2020). We previously found that NOX4 aggravated kidney injury by promoting ROS-mediated programmed cell death and inflammation (Wang, Yang 2020b, Meng et al. 2018). However, its regulatory mechanism in AAN is still unknown.

Macrophage infiltration is a hallmark of AAN and is believed to promote tubular epithelial cells (TEC) damage through the production of pro-inflammatory mediators to generate the reactive oxygen species in 
AAN (Allard et al. 2013, Wang et al. 2019, Pozdzik et al. 2010). In the current study of experimental AAN the prominent macrophage accumulation seen is consistent with studies of human AAN and previous studies of experimental AAN (Novitskaya et al. 2014, Lu et al. 2016). We found interstitial macrophage accumulation was associated with up-regulation of expression of the chemotactic molecules, CXCL12 and pro-inflammatory mediators, IL-1 $\beta$, which have been shown to promote monocyte recruitment in a several models of kidney disease. We also found that increased levels of MDA, production of lipid peroxidation, and decreased SOD and GSH, indicators of oxidative stress, after AA injection. In addition, protein level of NOX4 is increased in the pathogenesis of AAN in the kidneys of mice.

Aristolochic acid induced tubular damage and apoptosis is the primary insult in AAN. In the present study, we observed that AAl-treated mice displayed an increased number of TUNEL-stained cells with elevated protein levels of cleaved caspase-3 and KIM-1 in the kidneys.

Recent studies have revealed that EV may participate in renal tissue damage and regeneration through mediating inter-nephron communication. Bruno et al (Braun and Moeller 2015) reported incorporation of MSC-derived EV in acutely injured renal proximal tubular cells modulated the expressions of genes involved in cell recovery and repair by shuttling mRNA involved in transcription, proliferation, and immunoregulation. Eirin et al (Eirin et al. 2017) also found mesenchymal stem cell-derived extracellular vesicles attenuated kidney inflammation. Zhu et al (Zhu et al. 2019) reported in DN, high glucose led to increased excretion of exosomes from macrophages. Mesangial cells took up exosomes in vitro, which resulted in the activation and proliferation of mesangial cells and the secretion of extracellular matrix and inflammatory cytokines. In this study, we found in macrophage and tubular epithelial cells co-culture system, treatment with AAl up-regulated the protein levels of KIM-1 and NOX4 in TEC of the co-culture system. Interestingly,compared to AAl-induced TEC, the level of KIM-1 was significantly increased when AAl-induced TEC added to conditioned medium (CM) which comes from AAl-induced THP-1. In addition, AAl induces release of macrophages-derived EV. PHK67-labelled EV, which was isolated from primary macrophage of AAN, was injected into mice via renal in situ injection. Most importantly, AAl-induced, macrophage-derived EV enhanced the levels of KIM-1 and NOX-4, significantly promoted apoptosis in the kidney. In vivo and in vitro data suggest that macrophages infiltration promoted oxidative stress and programmed cell death of TEC is dependent on extracellular vesicles.

In our previous study, we demonstrated that LRG1 expression is significantly increased in patients with kidney injuries (Jiang et al. 2020b). Furthermore recent study highlighted the role of LRG1 in the progression of diabetic nephropathy (Zhang et al. 2020). It is suggested that LRG1 can promote completely different cellular processes including survival, proliferation, migration, and metastasis (Gao et al. 2020, Gu et al. 2020). However, the role of LRG1 in AAN remains largely unexplored. Our data support that KIM-1 and NOX-4 were upregulated in the presence of rhLRG1 in AAl-induced TEC. Furthermore, LRG1 expression in EV released from AAl-induced macrophage is significantly increased. Indeed, LRG1-enriched EV from macrophages promote renal injury and oxidative stress in AAl-induced mouse model. It was found LRG1 had a pivotal role in DN pathogenesis through TGF- $\beta$ signaling and was a risk factor for disease progression (Hong et al. 2019). We also found that LRG1-mediated TEC injury and oxidative 
stress is dependent on TGF $\beta R 1$ signaling. In contrast, knockdown TGF $\beta R 1$ of TEC, the expressions of KIM-1 and NOX-4 were decreased.

In summary, we identified EV as a potential link between macrophage-mediated inflammation and AAIinduced TEC oxidative stress and apoptosis (Fig. 10). TEC can take up macrophage-derived, LRG1enriched EV which likely play roles in controlling ANN progression. Given an important role of LRG1 in controlling oxidative stress, our findings that TGF $\beta$ 1 promotes LRG1-enriched EV uptake by upregulating NOX4 may provide different insights into the regulatory mechanisms of AAN, which may help design a better therapeutic strategy to treat AAN patients.

\section{Abbreviations}

AA, Aristolochic acid; AAN, Aristolochic acid nephropathy; EV, extracellular vesicles; TEC, tubular epithelial cells; $\mathrm{Cr}$, creatinine; BUN, blood urea nitrogen; NOX4, NADPH oxidase 4; CM, conditioned medium; LRG1, Leucine-rich a-2-glycoprotein 1; HK-2, human kidney tubular epithelial cells; mTEC, mouse kidney tubular epithelial cells; NTA, nanoparticle tracking analysis; TSG101, tumor susceptibility gene 101; ARF6, adenosine diphosphateribosyltion factor 6; PAS, periodic acid-Schiff; GME, glomerular mesangial expansion; IL, interleukin; CXCL, chemokine (C-X-C motif) ligand; Cyto D, Cytochalasin D

\section{Declarations}

\section{Author Contributions}

WJJ and CTX designed the study and performed experiments. CLD, JHD and CH performed animal study and generated the figures. TTM wrote the paper. All authors analyzed data, reviewed and edited the manuscript, and provided fundings.

\section{Fundings}

This work was supported by funding from National Nature Science Foundation of China (U19A2001, 82070628), University Natural Science Research Project of Anhui Province (KJ2019A0233), the University Synergy Innovation Program of Anhui Province (GXXT-2019-045囚GXXT-2020-063) and Natural Science Foundation of Anhui Province (2008085MH273). The authors thank the Center for Scientific Research of Anhui Medical University for valuable help in our experiment.

Ethics approval All protocols for animal experiments were approved by the Anhui Medical University Animal Care and Use Committee.

Consent to participate Not applicable

Consent for publication Not applicable

Conflicts of interest The authors declare no competing interests. 
Availability of data and material (data transparency) Not applicable

Code availability (software application or custom code) Not applicable

\section{References}

1. Allard T, Wenner T, Greten HJ, Efferth T. Mechanisms of herb-induced nephrotoxicity. Curr Med Chem. 2013; 20(22): 2812-9. http://doi.org/ 10.2174/0929867311320220006.

2. Braun GS, Moeller MJ. Progenitor cell-derived extracellular vesicles: an emerging diagnostic and therapeutic tool for renal disease. Nephrol Dial Transplant. 2015; 30(3): 339-41. http://doi.org/ 10.1093/ndt/gfv027.

3. Debelle FD, Vanherweghem JL, Nortier JL. Aristolochic acid nephropathy: a worldwide problem. Kidney Int. 2008; 74(2): 158-69. http://doi.org/ 10.1038/ki.2008.129.

4. Eirin A, Zhu XY, Puranik AS, Tang H, McGurren KA, van Wijnen AJ, et al. Mesenchymal stem cellderived extracellular vesicles attenuate kidney inflammation. Kidney Int. 2017; 92(1): 114-24. http://doi.org/ 10.1016/j.kint.2016.12.023.

5. Gao Y, Xie Z, Ho C, Wang J, Li Q, Zhang Y, et al. LRG1 Promotes Keratinocyte Migration and Wound Repair through Regulation of HIF-1alpha Stability. J Invest Dermatol 2020; 140(2): 455 - 64 e8. http://doi.org/10.1016/j.jid.2019.06.143.

6. Gladka MM. Cellular communication in a 'virtual lab': going beyond the classical ligand-receptor interaction. Cardiovasc Res. 2020; 116(7): e67-e9. http://doi.org/ 10.1093/cvr/cvaa076.

7. Gu Z, Xie D, Huang C, Ding R, Zhang R, Li Q, et al. MicroRNA-497 elevation or LRG1 knockdown promotes osteoblast proliferation and collagen synthesis in osteoporosis via TGF-beta1/Smads signalling pathway. J Cell Mol Med. 2020; 24(21): 12619-32. http://doi.org/ 10.1111/jcmm.15826.

8. Honarpisheh M, Foresto-Neto O, Steiger S, Kraft F, Koehler P, von Rauchhaupt E, et al. Aristolochic acid I determine the phenotype and activation of macrophages in acute and chronic kidney disease. Sci Rep. 2018; 8(1): 12169. http://doi.org/ 10.1038/s41598-018-30628-x.

9. Hong Q, Zhang L, Fu J, Verghese DA, Chauhan K, Nadkarni GN, et al. LRG1 Promotes Diabetic Kidney Disease Progression by Enhancing TGF-beta-Induced Angiogenesis. J Am Soc Nephrol. 2019; 30(4): 546-62. http://doi.org/ 10.1681/ASN.2018060599.

10. Jadot I, Decleves AE, Nortier J, Caron N. An Integrated View of Aristolochic Acid Nephropathy: Update of the Literature. Int J Mol Sci 2017; 18(2). http://doi.org/ 10.3390/ijms18020297.

11. Jiang $W, M a T$, Zhang $C$, Tang $X, X u Q$, Meng $X$, et al. Identification of urinary candidate biomarkers of cisplatin-induced nephrotoxicity in patients with carcinoma. J Proteomics. 2020a; 210: 103533. http://doi.org/ 10.1016/j.jprot.2019.103533.

12. Karpman D, Stahl AL, Arvidsson I. Extracellular vesicles in renal disease. Nat Rev Nephrol. 2017; 13(9): 545-62. http://doi.org/ 10.1038/nrneph.2017.98. 
13. Kim JY, Leem J, Jeon EJ. Protective Effects of Melatonin Against Aristolochic Acid-Induced Nephropathy in Mice. Biomolecules. 2019; 10(1). http://doi.org/10.3390/biom10010011.

14. Lu H, Bai Y, Wu L, Hong W, Liang Y, Chen B. Inhibition of Macrophage Migration Inhibitory Factor Protects against Inflammation and Matrix Deposition in Kidney Tissues after Injury. Mediators Inflamm. 2016; 2016: 2174682. http://doi.org/ 10.1155/2016/2174682.

15. Ma L, Shen Z, Hu H, Zhou H, Yu L, Jiang H, et al. Effects of rhein and Rheum palmatum L. extract on the pharmacokinetics and tissue distribution of aristolochic acid I and its demethylated metabolite in rats. J Ethnopharmacol. 2021;267:113537. http://doi.org/10.1016/j.jep.2020.113537.

16. Meng XM, Ren GL, Gao L, Yang Q, Li HD, Wu WF, et al. NADPH oxidase 4 promotes cisplatin-induced acute kidney injury via ROS-mediated programmed cell death and inflammation. Lab Invest 2018; 98(1): 63-78. http://doi.org/ 10.1038/labinvest.2017.120.

17. Novitskaya T, McDermott L, Zhang KX, Chiba T, Paueksakon P, Hukriede NA, et al. A PTBA small molecule enhances recovery and reduces postinjury fibrosis after aristolochic acid-induced kidney injury. Am J Physiol Renal Physiol. 2014; 306(5): F496-504. http://doi.org/ 10.1152/ajprenal.00534.2013.

18. Pozdzik AA, Berton A, Schmeiser HH, Missoum W, Decaestecker C, Salmon IJ, et al. Aristolochic acid nephropathy revisited: a place for innate and adaptive immunity? Histopathology. 2010;56(4):44963. http://doi.org/10.1111/j.1365-2559.2010.03509.x.

19. Pozdzik AA, Salmon IJ, Debelle FD, Decaestecker C, Van den Branden C, Verbeelen D, et al. Aristolochic acid induces proximal tubule apoptosis and epithelial to mesenchymal transformation. Kidney Int. 2008; 73(5): 595-607. http://doi.org/ 10.1038/sj.ki.5002714.

20. Ragot S, Saulnier PJ, Velho G, Gand E, de Hauteclocque A, Slaoui Y, et al. Dynamic Changes in Renal Function Are Associated With Major Cardiovascular Events in Patients With Type 2 Diabetes. Diabetes Care 2016; 39(7): 1259-66. http://doi.org/ 10.2337/dc15-2607.

21. Ren J, Rudemiller NP, Wen Y, Lu X, Privratsky JR, Crowley SD. The transcription factor Twist1 in the distal nephron but not in macrophages propagates aristolochic acid nephropathy. Kidney Int. 2020; 97(1): 119-29. http://doi.org/ 10.1016/j.kint.2019.07.016.

22. Rosenquist TA, Einolf HJ, Dickman KG, Wang L, Smith A, Grollman AP. Cytochrome P450 1A2 detoxicates aristolochic acid in the mouse. Drug Metab Dispos. 2010; 38(5): 761-8. http://doi.org/ 10.1124/dmd.110.032201.

23. Schmittgen TD, Livak KJ. Analyzing real-time PCR data by the comparative C(T) method. Nat Protoc. 2008; 3(6): 1101-8. http://doi.org/ 10.1038/nprot.2008.73.

24. Sedeek M, Nasrallah R, Touyz RM, Hebert RL. NADPH oxidases, reactive oxygen species, and the kidney: friend and foe. J Am Soc Nephrol. 2013; 24(10): 1512-8. http://doi.org/ 10.1681/ASN.2012111112.

25. Sundaresan NR, Gupta M, Kim G, Rajamohan SB, Isbatan A, Gupta MP. Sirt3 blocks the cardiac hypertrophic response by augmenting Foxo3a-dependent antioxidant defense mechanisms in mice. J Clin Invest. 2009; 119(9): 2758-71. http://doi.org/ 10.1172/JCI39162. 
26. Susnik N, Sorensen-Zender I, Rong S, von Vietinghoff S, Lu X, Rubera I, et al. Ablation of proximal tubular suppressor of cytokine signaling 3 enhances tubular cell cycling and modifies macrophage phenotype during acute kidney injury. Kidney Int 2014; 85(6): 1357-68. http://doi.org/ 10.1038/ki.2013.525.

27. Tang TT, Lv LL, Lan HY, Liu BC. Extracellular Vesicles: Opportunities and Challenges for the Treatment of Renal Diseases. Front Physiol. 2019; 10: 226. http://doi.org/ 10.3389/fphys.2019.00226.

28. Thery C, Amigorena S, Raposo G, Clayton A. Isolation and characterization of exosomes from cell culture supernatants and biological fluids. Curr Protoc Cell Biol. 2006; Chap. 3: Unit 322. http://doi.org/ 10.1002/0471143030.cb0322s30.

29. Tkach M, Thery C. Communication by Extracellular Vesicles: Where We Are and Where We Need to Go. Cell 2016; 164(6): 1226-32. http://doi.org/ 10.1016/j.cell.2016.01.043.

30. van Niel G, D'Angelo G, Raposo G. Shedding light on the cell biology of extracellular vesicles. Nat Rev Mol Cell Biol. 2018; 19(4): 213-28. http://doi.org/ 10.1038/nrm.2017.125.

31. Wang JN, Yang Q, Yang C, Cai YT, Xing T, Gao L, et al. Smad3 promotes AKI sensitivity in diabetic mice via interaction with p53 and induction of NOX4-dependent ROS production. Redox Biol. 2020b; 32: 101479. http://doi.org/ 10.1016/j.redox.2020.101479.

32. Wang L, Liu N, Xue X, Zhou S. The Effect of Overexpression of the Enhancer of Zeste Homolog 1 (EZH1) Gene on Aristolochic Acid-Induced Injury in HK-2 Human Kidney Proximal Tubule Cells In Vitro. Med Sci Monit. 2019; 25: 801-10. http://doi.org/ 10.12659/MSM.911611.

33. Wang X, Xue N, Zhao S, Shi Y, Ding X, Fang Y. Upregulation of miR-382 contributes to renal fibrosis secondary to aristolochic acid-induced kidney injury via PTEN signaling pathway. Cell Death Dis 2020a; 11(8): 620. http://doi.org/ 10.1038/s41419-020-02876-1.

34. Wu TK, Wei CW, Pan YR, Cherng SH, Chang WJ, Wang HF, et al. Vitamin C attenuates the toxic effect of aristolochic acid on renal tubular cells via decreasing oxidative stressmediated cell death pathways. Mol Med Rep. 2015; 12(4): 6086-92. http://doi.org/ 10.3892/mmr.2015.4167.

35. Yaribeygi H, Farrokhi FR, Rezaee R, Sahebkar A. Oxidative stress induces renal failure: A review of possible molecular pathways. J Cell Biochem. 2018; 119(4): 2990-8. http://doi.org/ $10.1002 /$ jcb.26450.

36. Yoo JY, Cha DR, Kim B, An EJ, Lee SR, Cha JJ, et al. LPS-Induced Acute Kidney Injury Is Mediated by Nox4-SH3YL1. Cell Rep. 2020; 33(3): 108245. http://doi.org/ 10.1016/j.celrep.2020.108245.

37. Zeng Y, Zheng L, Yang Z, Yang C, Zhang Y, Li J, et al. Protective effects of cyclic helix B peptide on aristolochic acid induced acute kidney injury. Biomed Pharmacother. 2017; 94: 1167-75. http://doi.org/ 10.1016/j.biopha.2017.07.131.

38. Zhang A, Fang H, Chen J, He L, Chen Y. Role of VEGF-A and LRG1 in Abnormal Angiogenesis Associated With Diabetic Nephropathy. Front Physiol. 2020; 11: 1064. http://doi.org/ 10.3389/fphys.2020.01064. 
39. Zhang HM, Zhao XH, Sun ZH, Li GC, Liu GC, Sun LR, et al. Recognition of the toxicity of aristolochic acid. J Clin Pharm Ther. 2019; 44(2): 157-62. http://doi.org/ 10.1111/jcpt.12789.

40. Zhang PL, Liu ML. Extracellular vesicles mediate cellular interactions in renal diseases-Novel views of intercellular communications in the kidney. J Cell Physiol. 2021. http://doi.org/ 10.1002/jcp.30268.

41. Zhu QJ, Zhu M, Xu XX, Meng XM, Wu YG. Exosomes from high glucose-treated macrophages activate glomerular mesangial cells via TGF-beta1/Smad3 pathway in vivo and in vitro. FASEB J. 2019; 33(8): 9279-90. http://doi.org/ 10.1096/fj.201802427RRR.

\section{Figures}




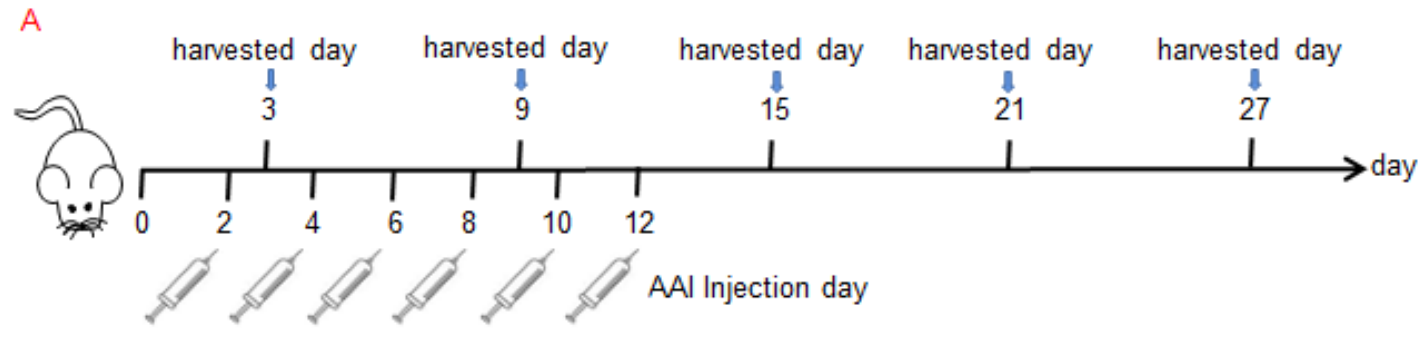

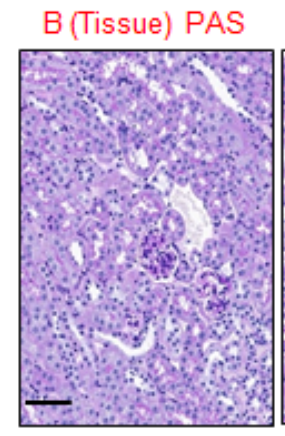

(day) 0

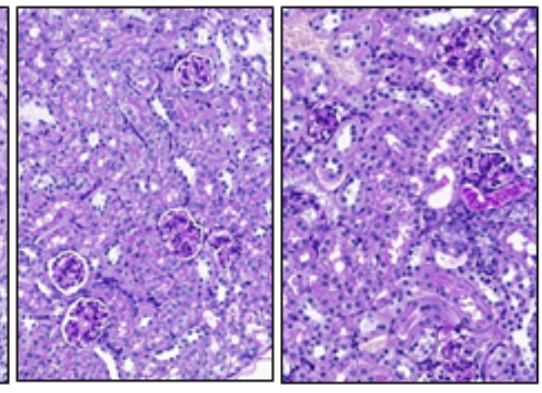

3

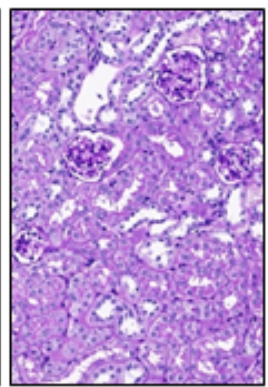

15

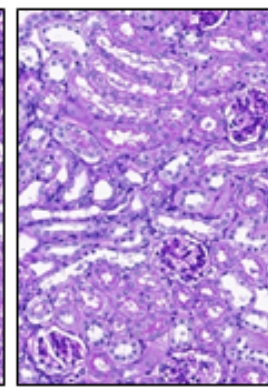

21

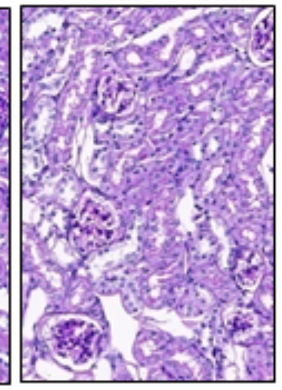

27
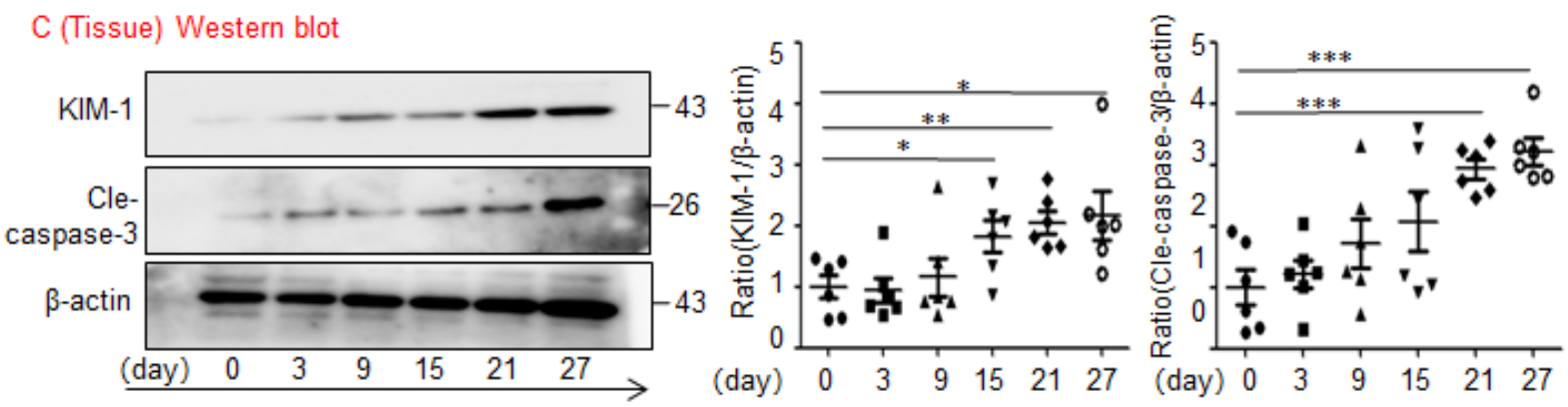

D (Tissue) TUNEL

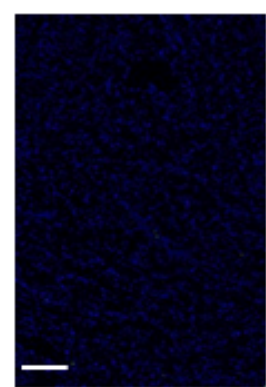

(day) 0

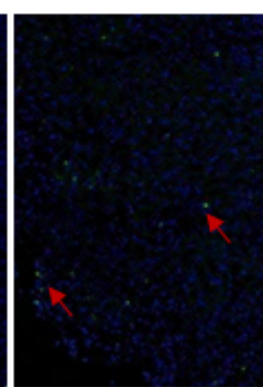

3

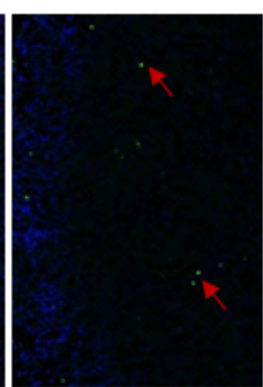

9

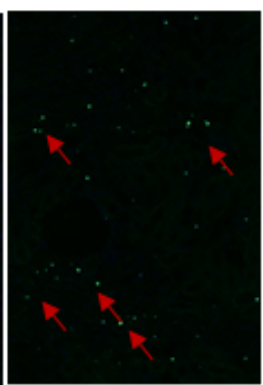

15

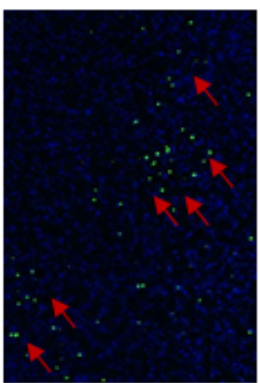

21

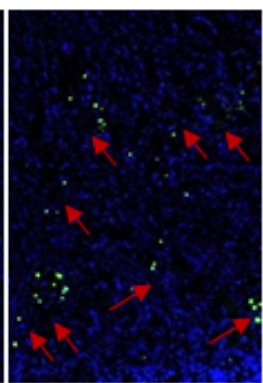

$\stackrel{27}{\longrightarrow}$

\section{Figure 1}

Aristolochic acid induces kidney injury in a time-dependent manner (A) AAN model was established. (B) PAS staining of kidney sections. Scale bar, $100 \mu \mathrm{m}$. (C) Western blot was performed on different treatment group to determine KIM-1 and cleaved caspase-3 expressions. Dot plots show the corresponding quantification of band intensity and fold change. (D) TUNEL staining of kidney sections. Scale bar, 
$200 \mu \mathrm{m}$. Similar results were obtained in 3 independent experiments with 10 mice per group. ${ }^{\star \star \star} \mathrm{P}<0.001$, **P<0.01, *P<0.05.
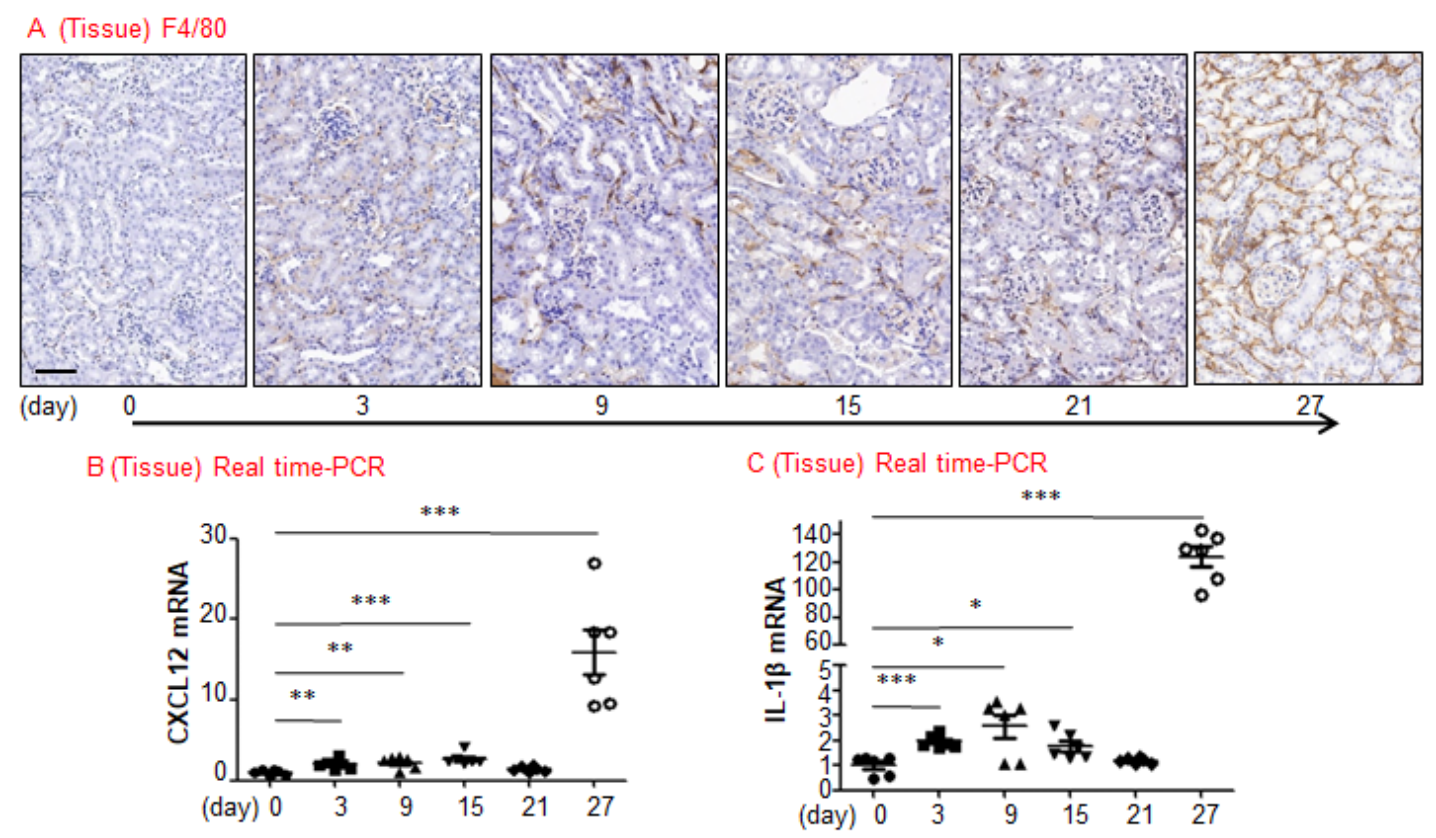

D
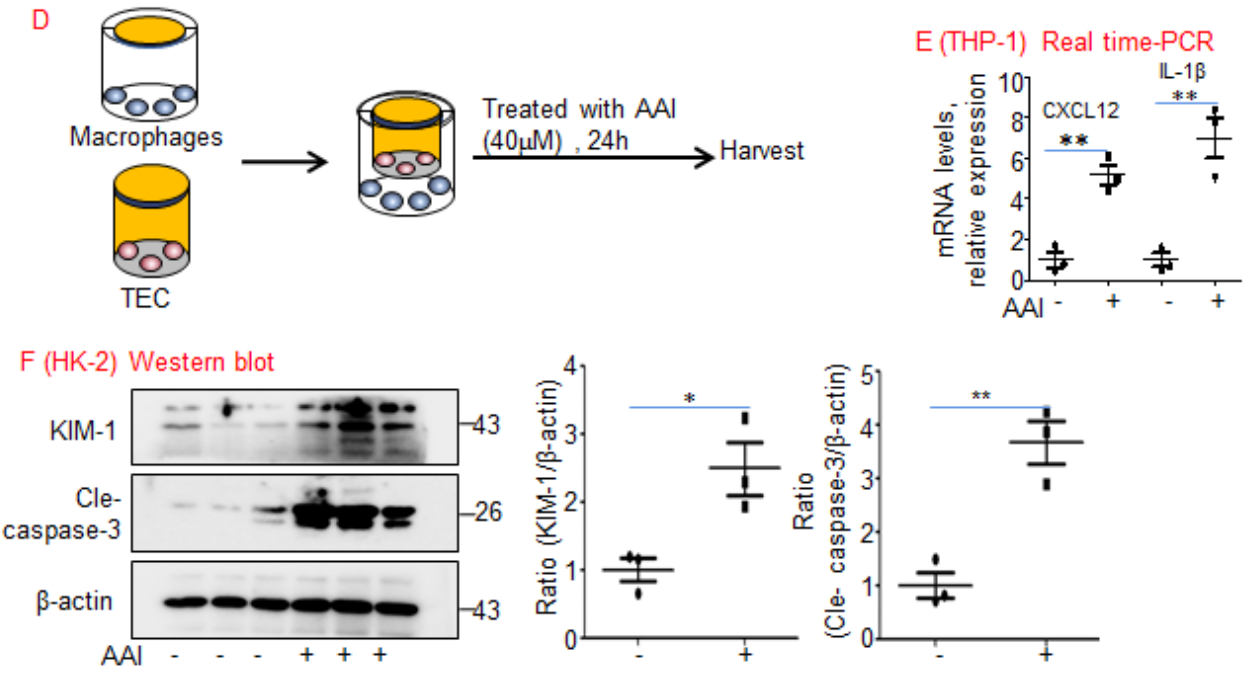

G(HK-2) Flow cytometry
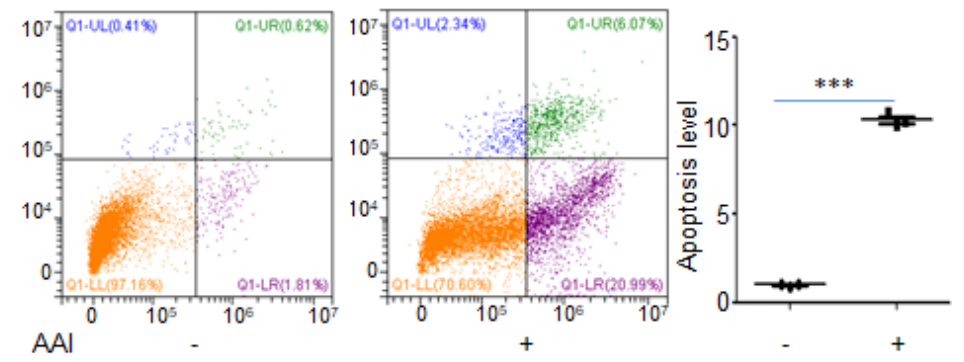

\section{Figure 2}

Macrophage infiltration promotes AAl-induced injury (A) F4/80 staining of kidney sections. Scale bar, $100 \mu \mathrm{m}$. (B) CXCL12 mRNA was assessed by real-time qPCR. Dot plots show the corresponding quantification of band intensity and fold change. (C) IL-1 $\beta$ mRNA was assessed by real-time qPCR. Dot 
plots show the corresponding quantification of band intensity and fold change. (D) TECs and macrophages co-culture system. (E) The mRNA levels of CXCL12 and IL-1 $\beta$ in THP-1 were assessed by real-time qPCR. Dot plots show the corresponding quantification of band intensity and fold change. $(F)$ Western blot analysis showing the protein levels of KIM-1 and cleaved caspase-3. Dot plots show the corresponding quantification of band intensity and fold change. (G) Flow cytometry assay of HK-2 cells. Dot plots show the corresponding quantification of band intensity and fold change. Similar results were obtained in 3 independent experiments with 10 mice per group or in triplicate culture assays. ${ }^{* \star \star P} P<0.001$, $\star * P<0.01, * P<0.05$. 


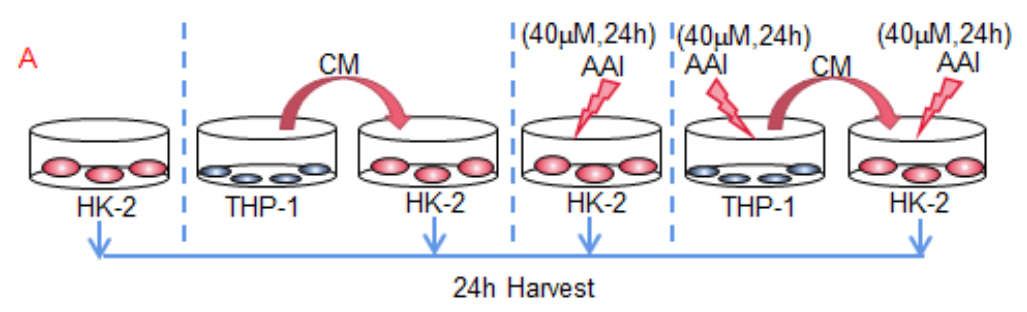

B (THP-1) Real time-PCR

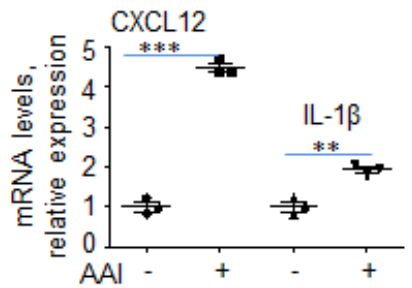

C (HK-2) Western blot
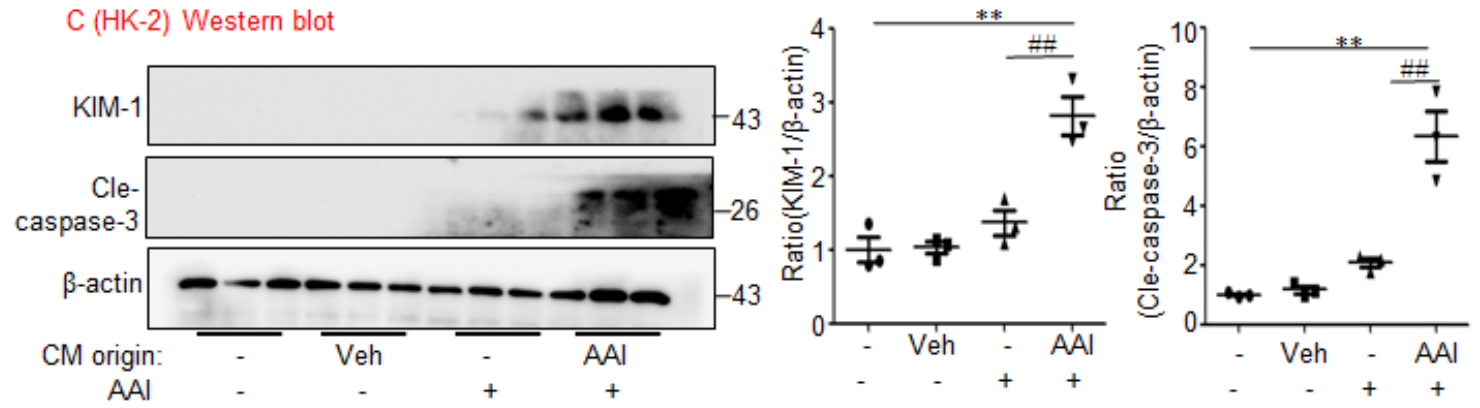

D (HK-2) Immunofluorescence
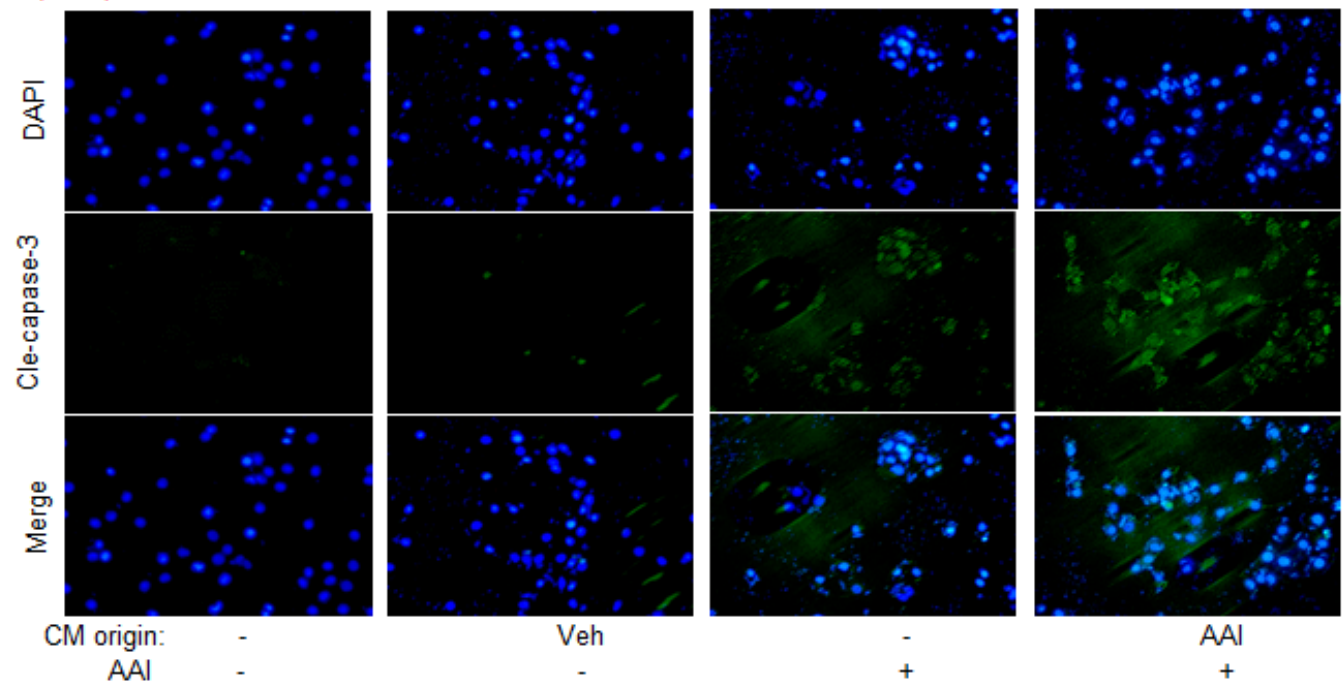

Veh
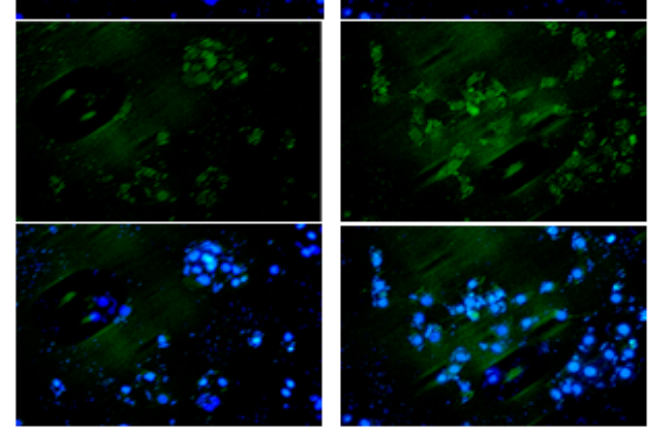

AAl

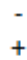

AAl

E (HK-2) Flow cytometry

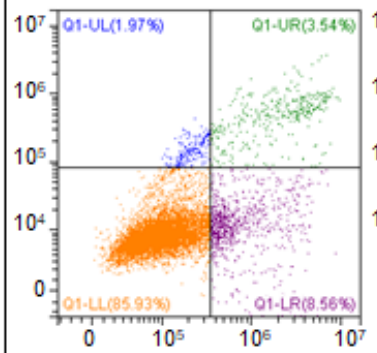

$\mathrm{CM}$ origin:
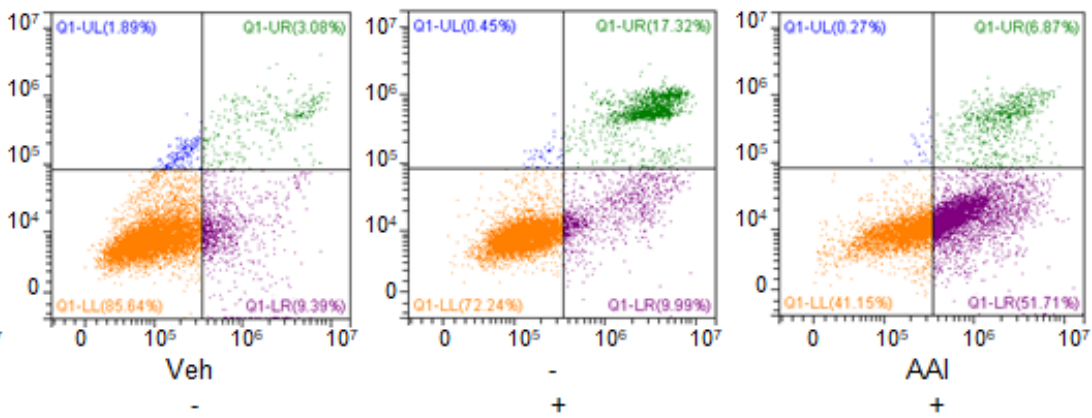

\section{Figure 3}

THP-1 conditioned media promotes AAl-induced HK-2 injury (A) THP-1/HK-2 conditioned media culture model. (B) The mRNA levels of CXCL12 and IL-1 $\beta$ in THP-1 was assessed by real-time qPCR. (C) Western blot analysis showing the protein expression of KIM-1and cleaved caspase-3 in HK-2 cells. Dot plots show the corresponding quantification of band intensity and fold change. (D) Representative images of cleaved caspase-3 immunohistochemical staining in HK-2 cells. Scale bar, 50 $\mu \mathrm{m}$. (E) Flow cytometry 
assay of HK-2. Dot plots show the corresponding quantification of band intensity and fold change. Similar results were obtained in triplicate culture assays. ${ }^{*} * P<0.001,{ }^{*} P<0.01, \# \# P<0.01$

A (THP-1) NTA

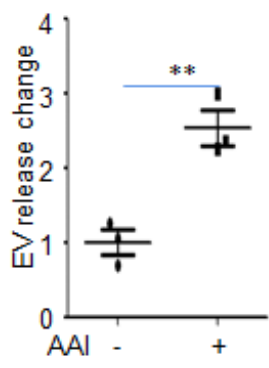

B (THP-1) Electron microscopy

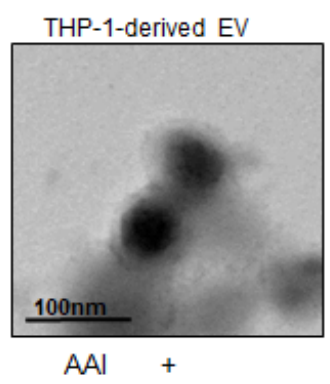

C (THP-1) Western blot

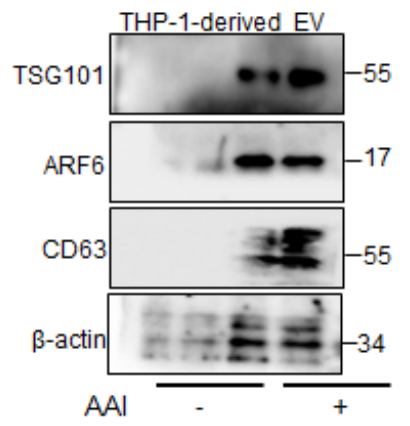

D (BMDM) NTA

E (BMDM) Western blot
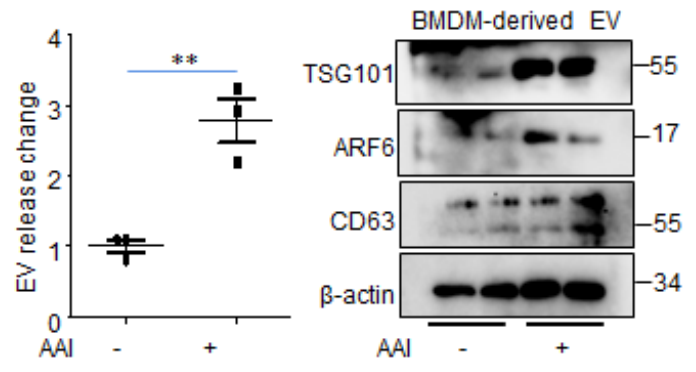

$\mathrm{F}$

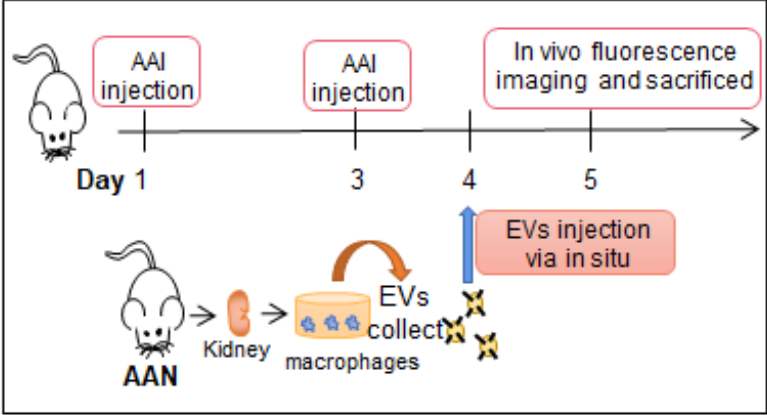

G
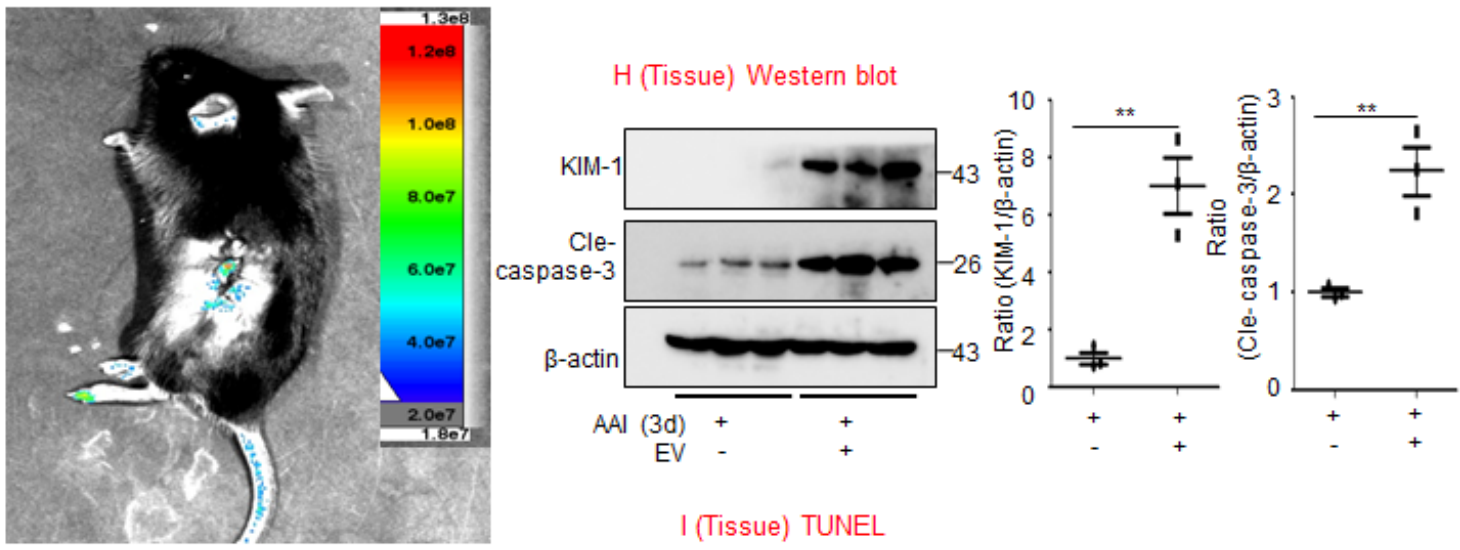

I (Tissue) TUNEL
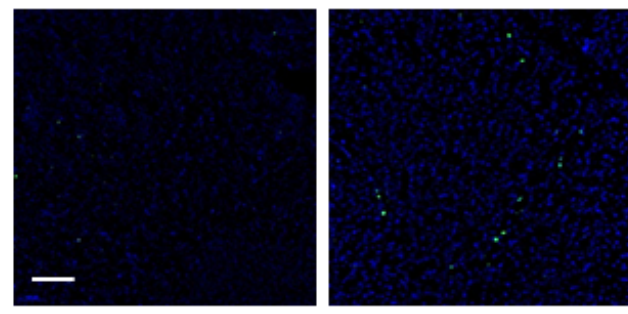

AAl (3d)
EV -

\section{Figure 4}

Macrophages-derived EV promote AAl-induced injury (A) Representative image of NTA showing the concentration of THP-1-derived EVs. Dot plots show the corresponding quantification of band intensity and fold change. (B) Transmission electron photomicrographs of THP-1-derived EVs. Scale bar, 100nm. 
(C) Immunoblot for EV markers, TSG101, ARF6, CD63 and $\beta$-action, in THP-1-derived EVs. (D) Representative image of NTA showing the concentration of BMDM-derived EVs. Dot plots show the corresponding quantification of band intensity and fold change. (E) Immunoblot for EV markers, TSG101, ARF6, CD63 and $\beta$-action, in BMDM-derived EVs. (F) Purified EVs were isolated from primary macrophage of AAN and PHK67-labelled EVs were injected into mice via renal in situ injection. (G) Imaging of PKH67labeled EVs in mice. About $100 \mu \mathrm{g}$ (at protein level) in $100 \mu \mathrm{L}$ exosomes from AAl exposured mice primary renal macrophage, labeled with PKH67 were injected via in situ. About $24 \mathrm{~h}$ after the in situ injection, in vivo fluorescence imaging were performed. $(\mathrm{H})$ Western blot analysis showing the protein expression of KIM-1 and cleaved caspase-3. Dot plots show the corresponding quantification of band

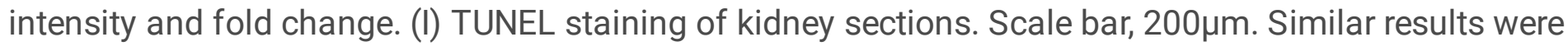
obtained in 3 independent experiments with 10 mice per group or in triplicate culture assays. ${ }^{\star \star} P<0.01$. 

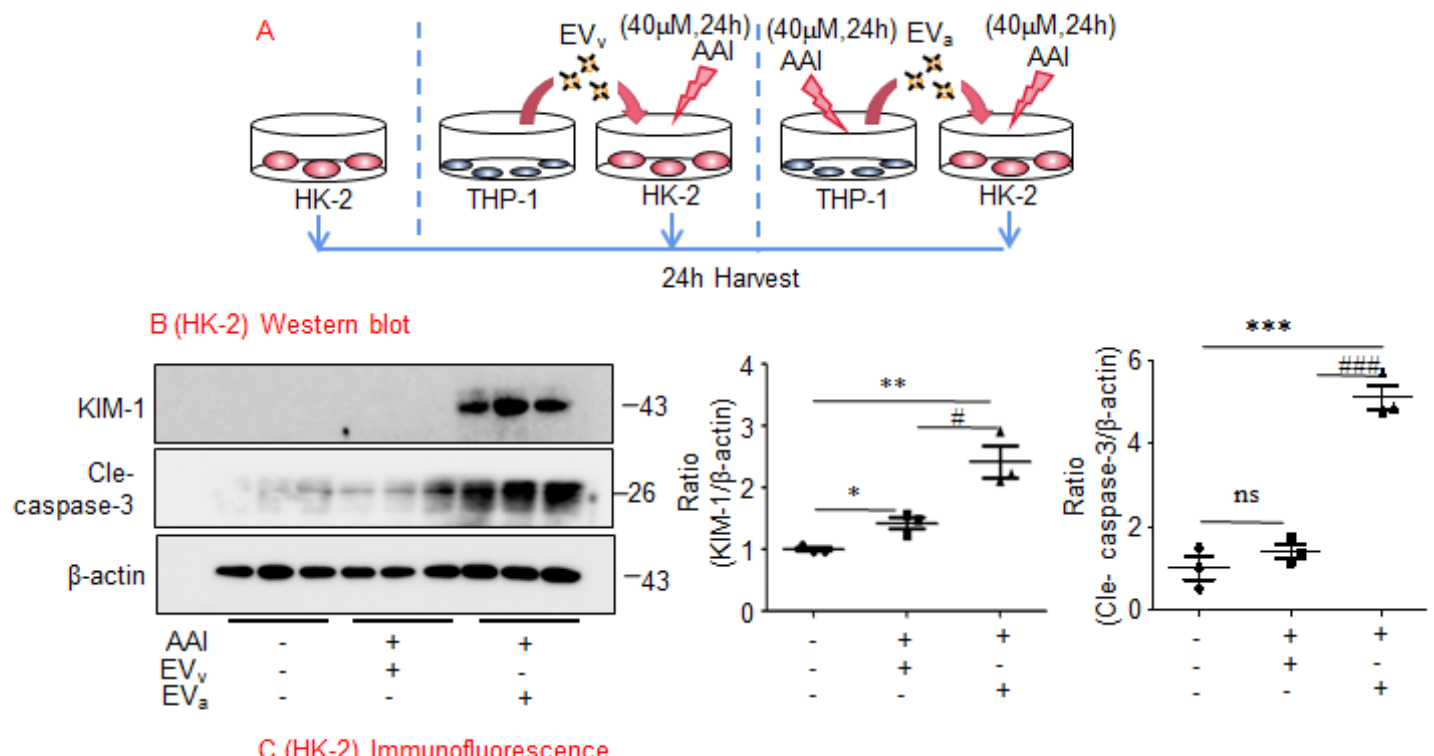

C (HK-2) Immunofluorescence
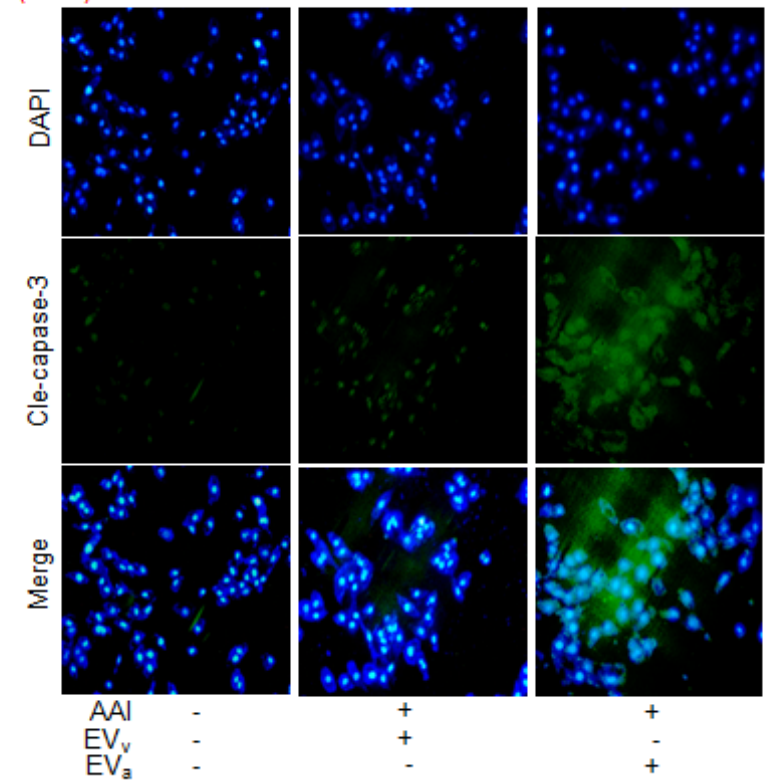

D (HK-2) Flow cytometry
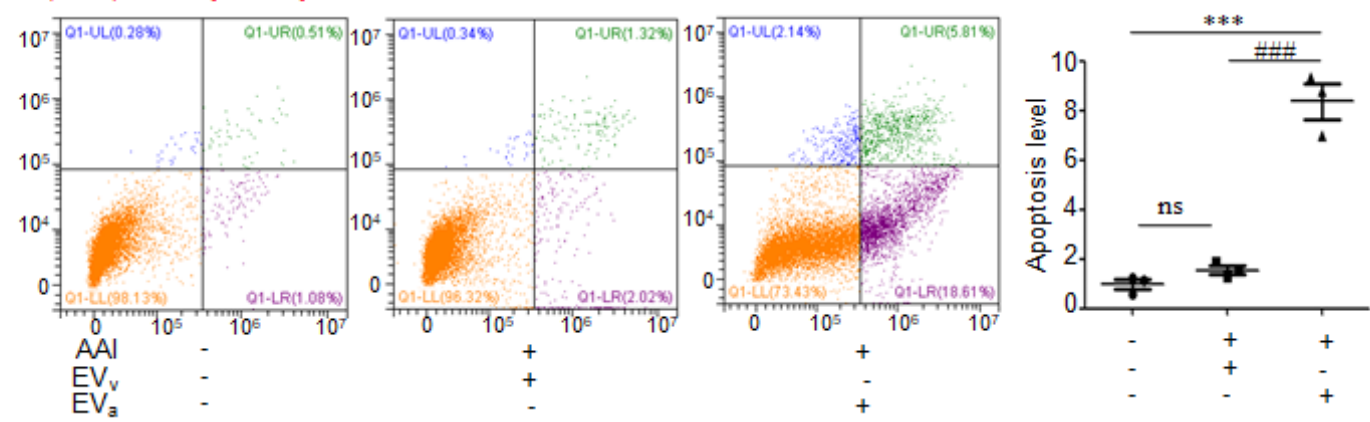

\section{Figure 5}

AAl-treated, THP-1-derived EV promote AAl-induced HK-2 cells injury (A) The cell model was established to investigate the role of EV. EVV was derived from THP-1 and incubated with AAl-treated HK-2. EVa was derived from AAl-treated THP-1 and incubated with AAl-treated HK-2. (B) Western blot analysis showing the protein expression of KIM-1 and cleaved caspase-3 in different group. Dot plots show the corresponding quantification of band intensity and fold change. (C) Representative images of cleaved 
caspase-3 immunofluorescence staining in different group. Scale bar, 50 $\mu \mathrm{m}$. (D) Flow cytometry assay of HK-2 cell. Dot plots show the corresponding quantification of band intensity and fold change. Similar results were obtained in triplicate culture assays. ${ }^{\star} \star * P<0.001$, ${ }^{\star *} P<0.01, \# \# \# P<0.001, \# \# P<0.01$.

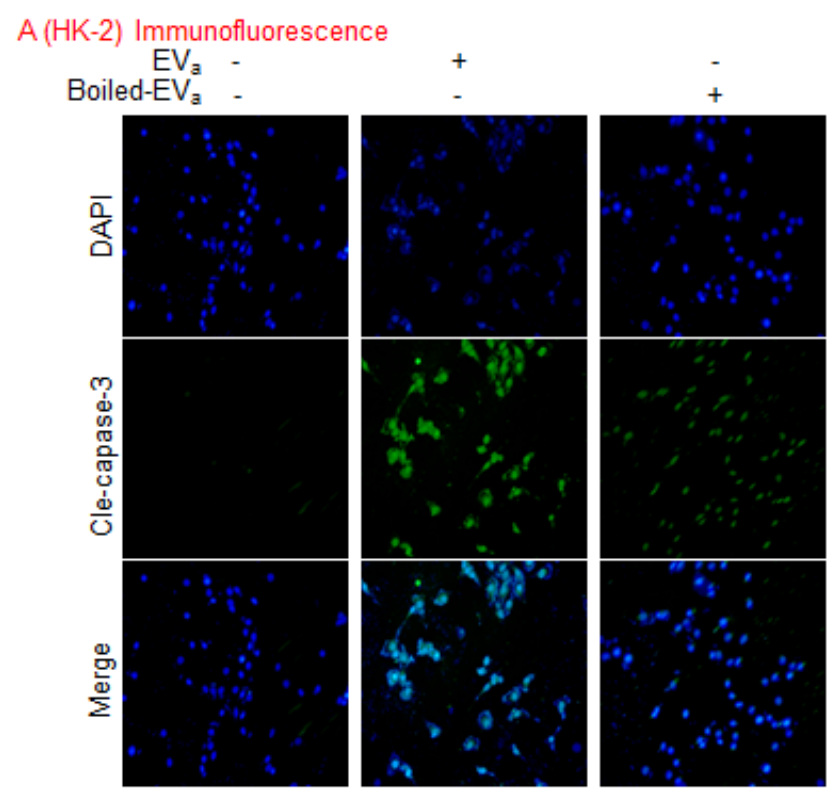

B (HK-2) confocal microscopy

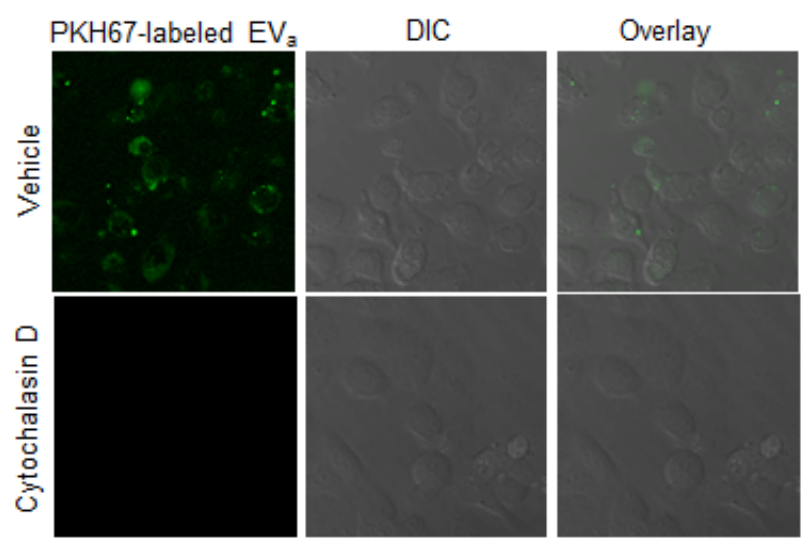

C (HK-2) Western blot
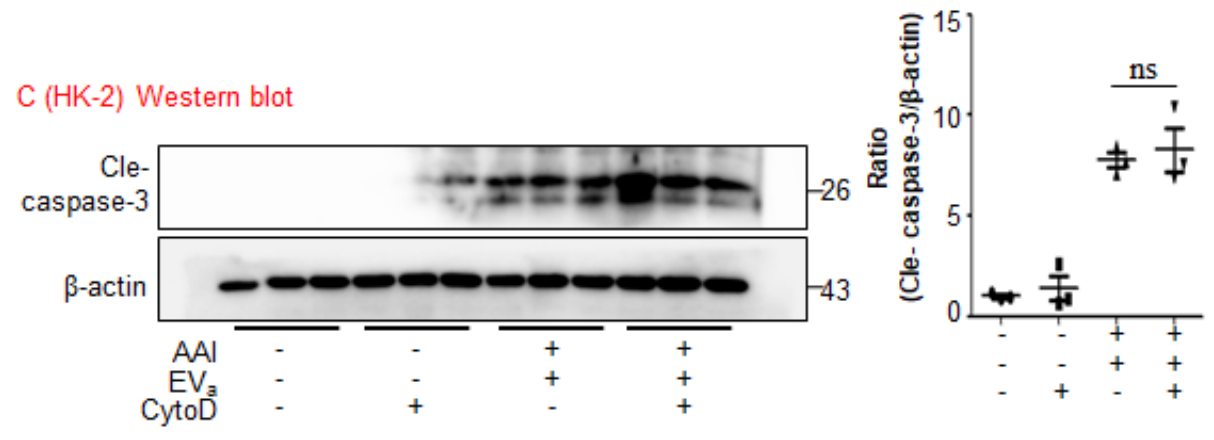

Figure 6

macrophages-derived EV promotes AAl-induced injury at the level of the plasma membrane (A) HK-2 cells were incubated with EV isolated from AAl-treated THP-1 cells were either intact or boiled at $95^{\circ} \mathrm{C}$ for 20 
min. Representative images of cleaved caspase-3 immunofluorescence staining in different group. Scale bar, $50 \mu \mathrm{m}$. (B) HK-2 cells were pretreated with cytochalasin D $(1 \mu \mathrm{M})$ followed by 2 hour incubation with AAl-treated, THP-1-derived EVa $(1010 / \mathrm{ml})$. Prior to this incubation, EVa were labeled using fluorescent dye PKH67. EVa uptake by HK-2 was visualized using confocal microscopy and quantified. (C) Western blot analysis showing the protein expression of cleaved caspase-3 in different group. Dot plots show the corresponding quantification of band intensity and fold change. Similar results were obtained in triplicate culture assays.
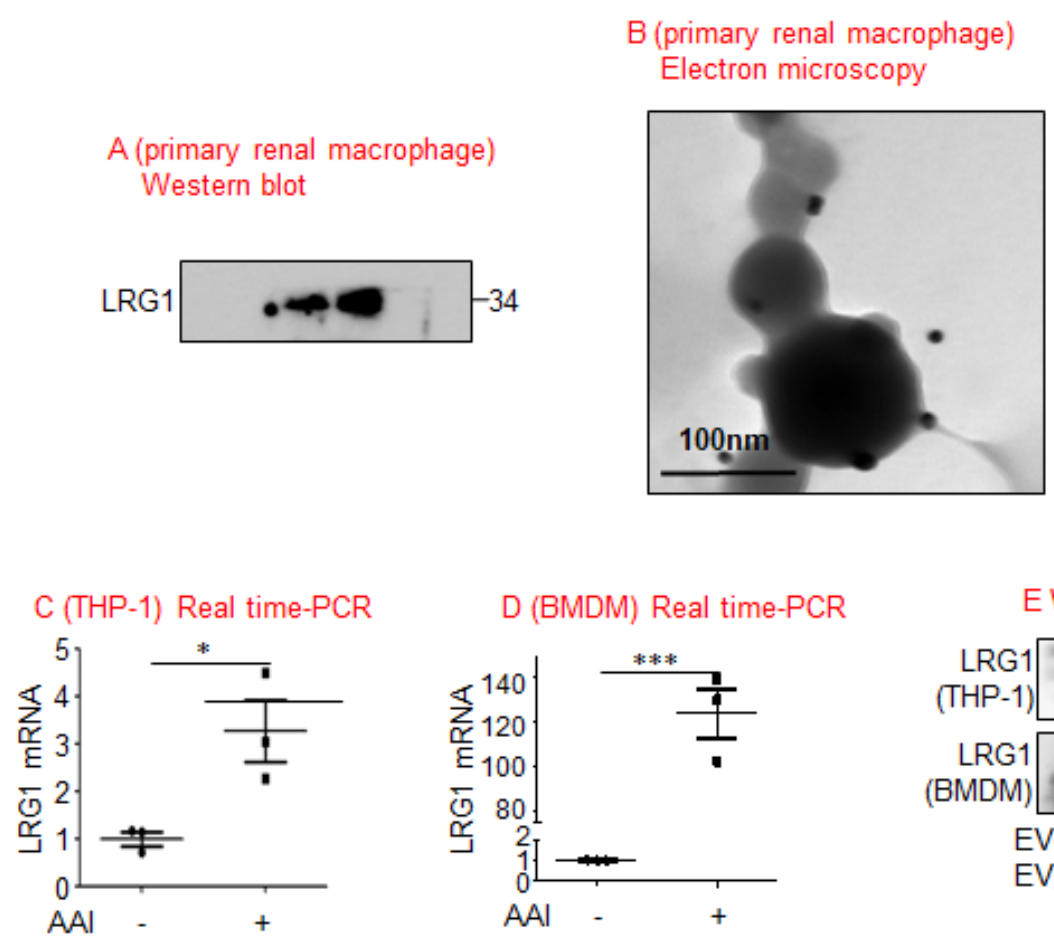

EWestern blot
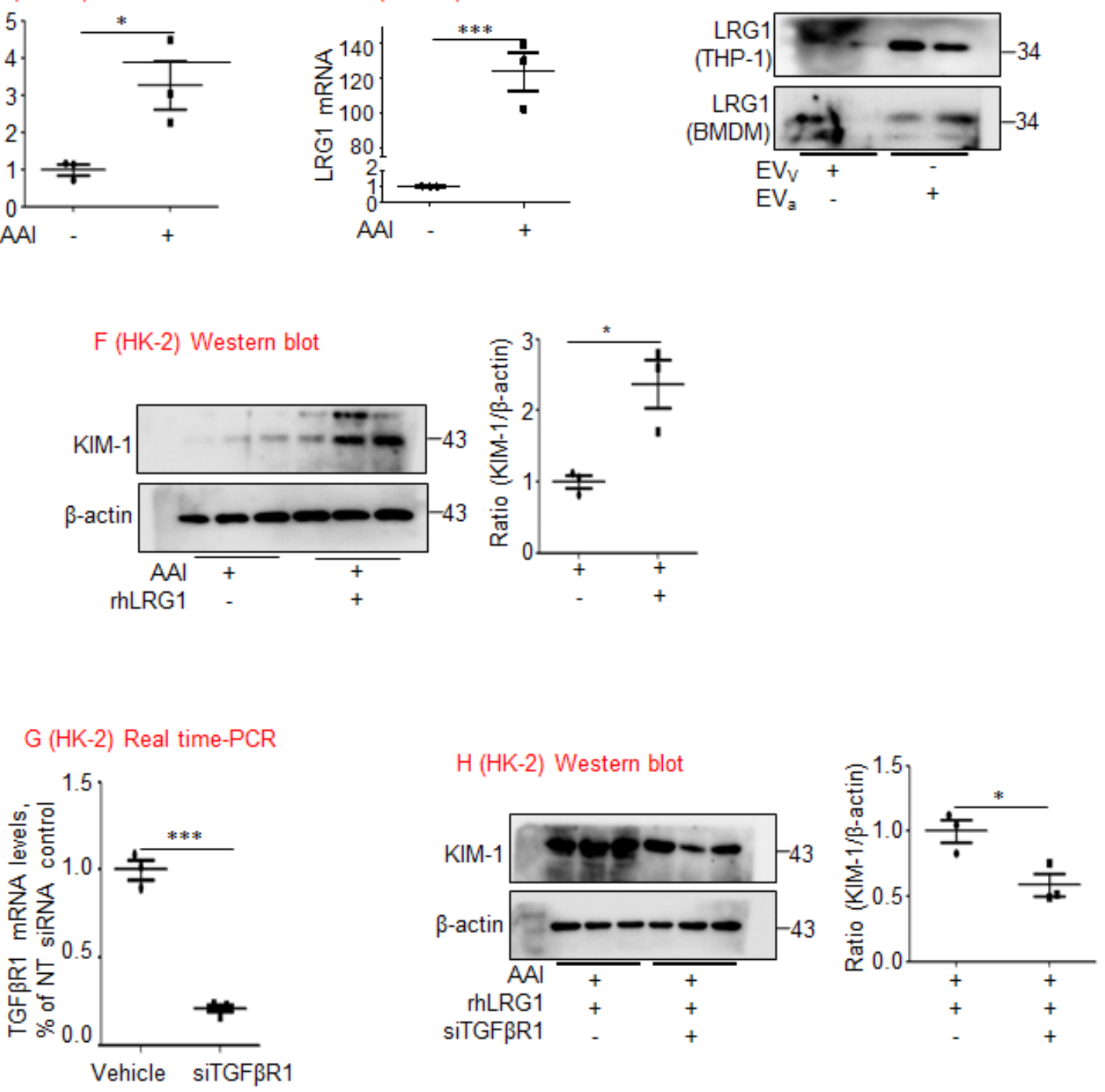


\section{Figure 7}

Macrophages-derived, LRG1-enriched EV promoting TECs injury is TGF $\beta R 1$-dependent (A) The protein level of LRG1 in EV derived from primary renal macrophage cells of AAN mice model. (B) Representative transmission electron photomicrographs immunogold- labeled with an anti-LRG1 antibody of primary renal macrophage-derived EV of AAN. Scale bar: 100nm. (C) LRG1 mRNA was assessed by real-time qPCR in THP-1. Dot plots show the corresponding quantification of band intensity and fold change. (D) LRG1 mRNA was assessed by real-time qPCR in BMDM. Dot plots show the corresponding quantification of band intensity and fold change. (E) Different protein level of LRG1 in EV derived from AAl-induced THP-1and BMDM. (F) The protein levels of KIM-1 in HK-2 cells stimulated with AAl and treated with human recombinant LRG1( $\mathrm{rhLRG} 1)(10 \mathrm{ng} / \mathrm{ml})$. Dot plots show the corresponding quantification of band intensity and fold change. (G) The level of TGF $\beta R 1$ in HK-2 cells treated with siRNA-TGF $\beta R 1$ for 24 hours. $(\mathrm{H})$ The protein levels of KIM-1 in LRG1-pretreated, AAl-induced HK-2 cells treated with siRNA-TGFBR1. Dot plots show the corresponding quantification of band intensity and fold change. Similar results were obtained in 3 independent experiments with 10 mice per group or in triplicate culture assays. ${ }^{* \star *} \mathrm{P}<0.001$, $\star * P<0.01, * P<0.05$. 

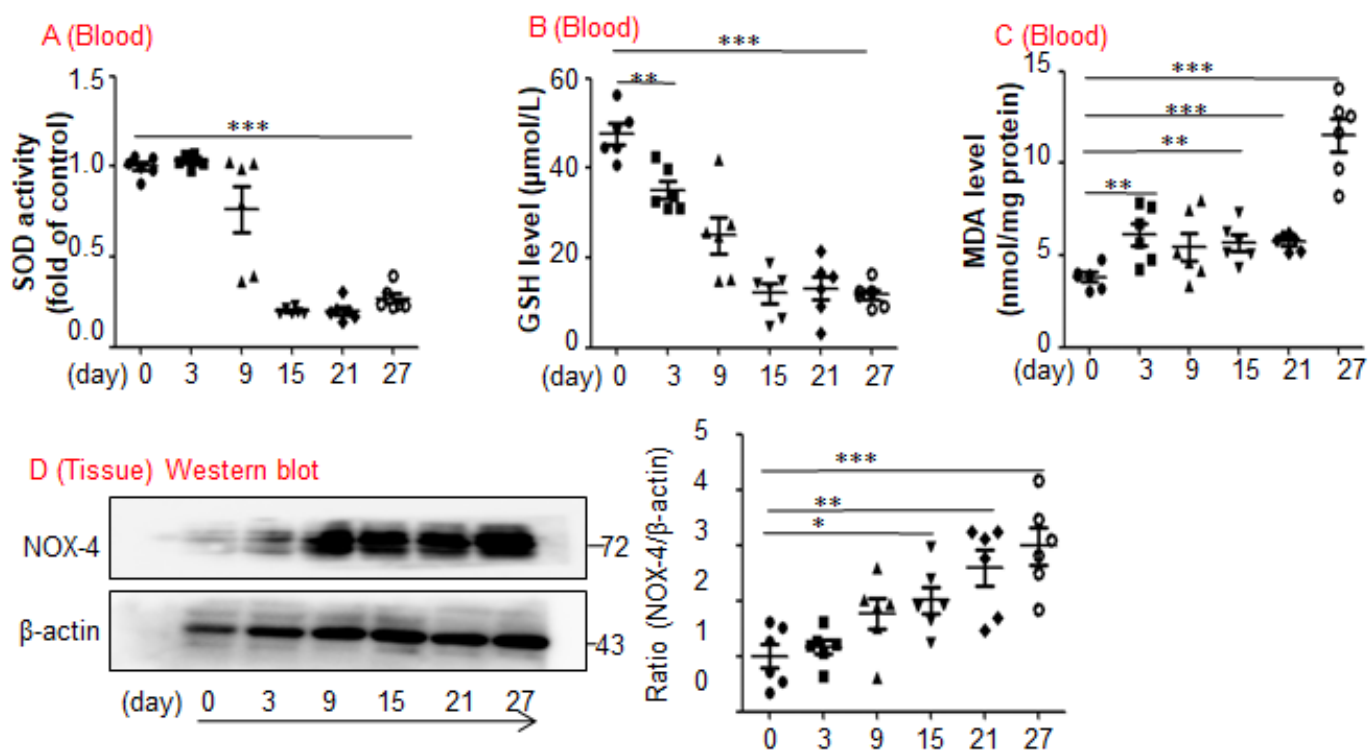

E (HK-2) Western blot
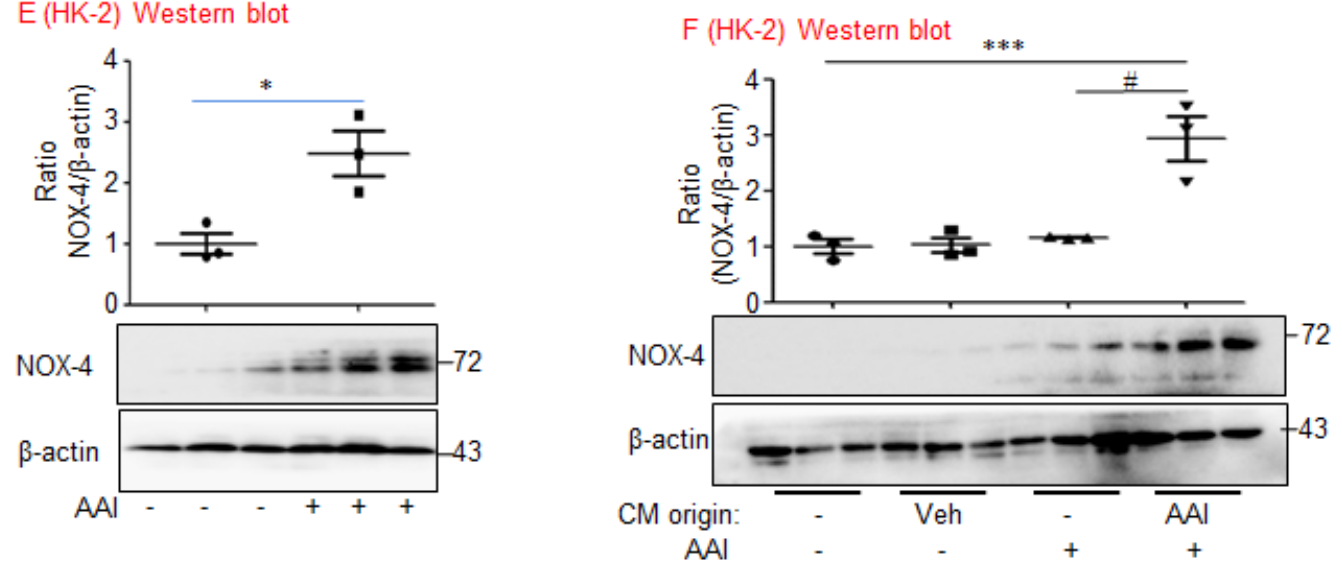

G (Tissue) Western blot
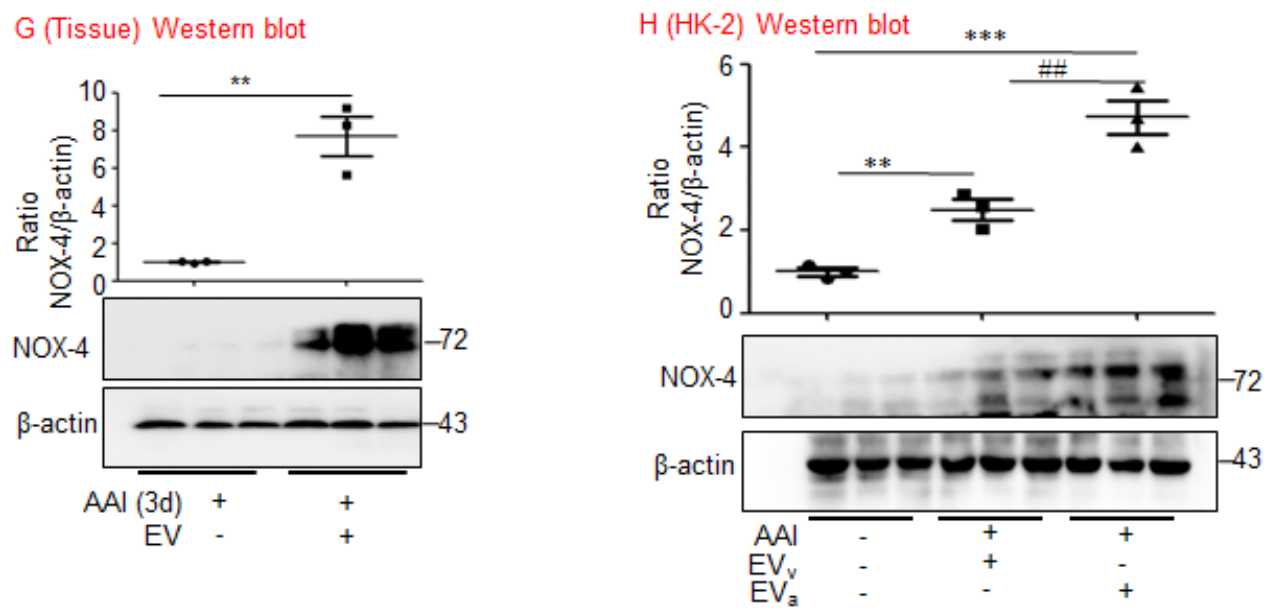

\section{Figure 8}

macrophages-derived EV promotes NOX4 expression (A) Level of SOD was assessed in different group. (B) Level of GSH was assessed in different group. (C) Level of MDA was assessed in different group. (D) Western blot was performed on different treatment group to determine NOX4 expression. Dot plots show the corresponding quantification of band intensity and fold change. (E) Western blot analysis showing the protein levels of NOX4 in THP-1/HK-2 co-culture system. Dot plots show the corresponding 
quantification of band intensity and fold change. (F) Western blot analysis showing the protein levels of NOX4 in THP-1/HK-2 conditioned media culture model. Dot plots show the corresponding quantification of band intensity and fold change. $(G)$ Western blot analysis showing the protein expression of NOX4 in macrophages-derived EV, isolated from primary macrophages of AAI-induced, 27-day AAN model mice kidney tissues, injected into AAl-induced, 3 -day mice. Dot plots show the corresponding quantification of band intensity and fold change. $(\mathrm{H})$ Western blot analysis showing the protein expression of NOX4 in AAlinduced HK-2 cells with addition of AAl-treated, THP-1-derived EV. Dot plots show the corresponding quantification of band intensity and fold change. Similar results were obtained in 3 independent experiments with 10 mice per group or in triplicate culture assays. ${ }^{* \star *} P<0.001,{ }^{*} * P<0.01,{ }^{*} P<0.05$. $\# \# \mathrm{P}<0.01$. 

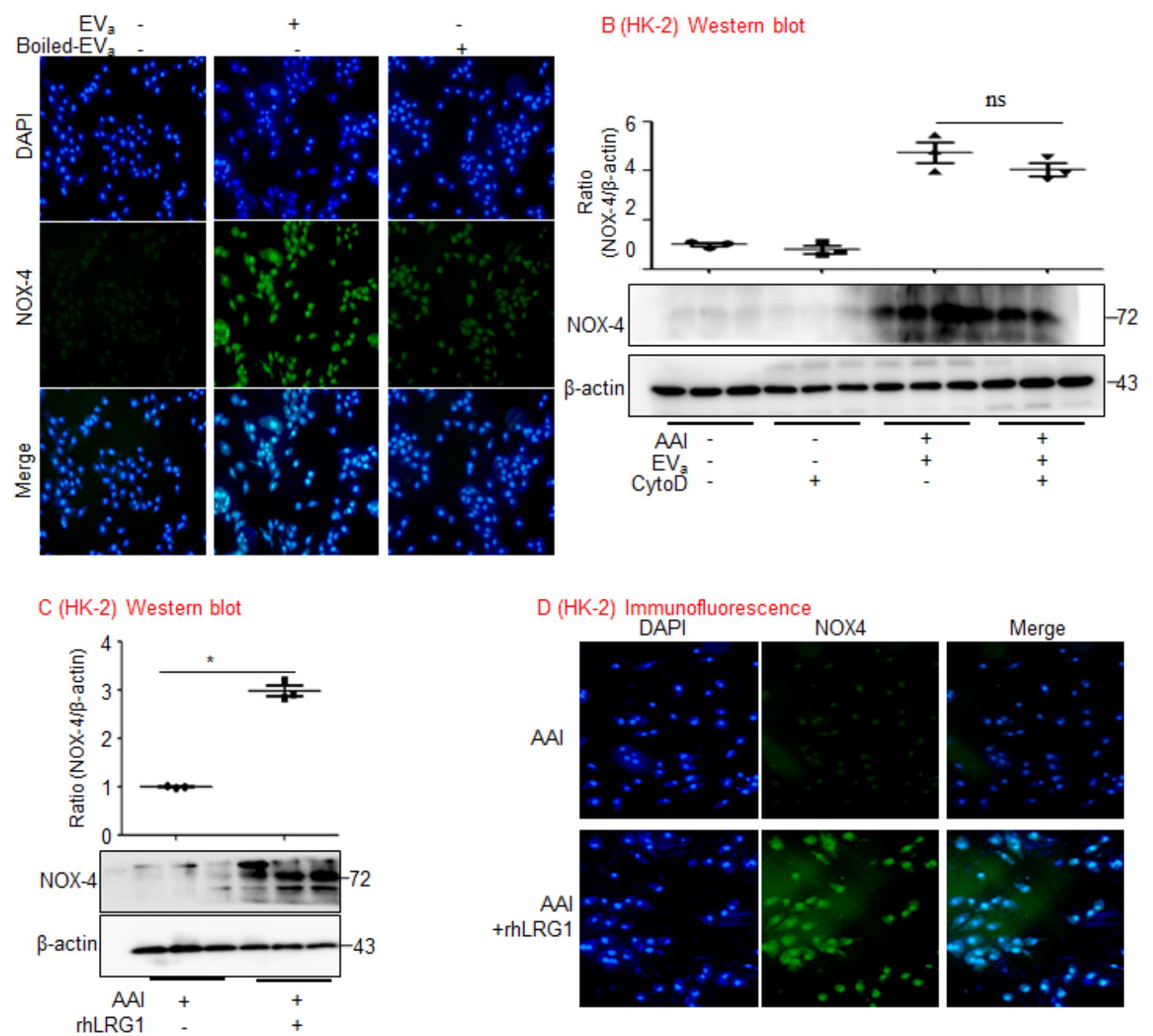

$\mathrm{E}(\mathrm{HK}-2)$ Western blot

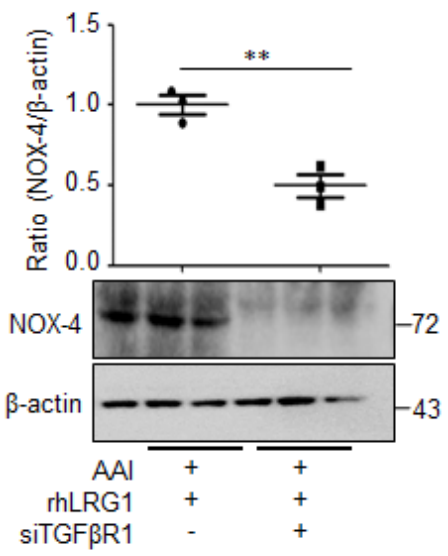

F (HK-2) Immunofluorescence

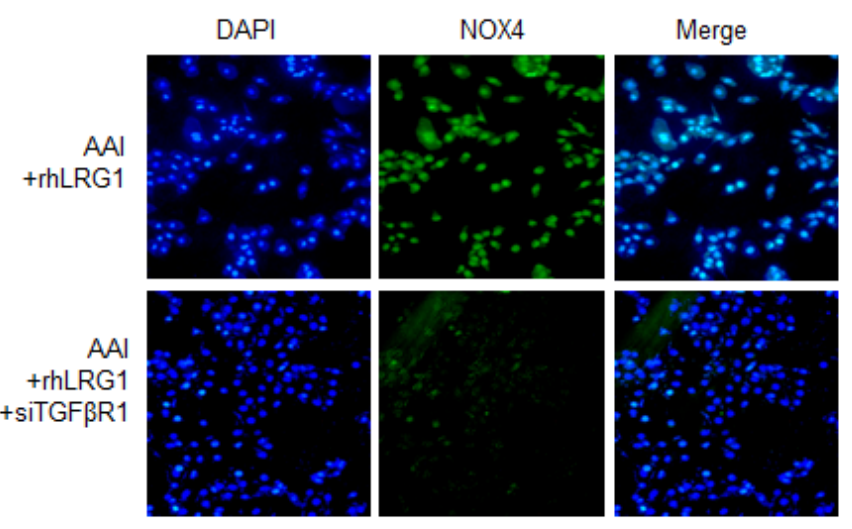

\section{Figure 9}

Macrophages-derived, LRG1-enriched EV mediating TEC injury by upregulating NOX4 (A) HK-2 cells were incubated with EV isolated from AAl-treated THP-1 cells were either intact or boiled at $95^{\circ} \mathrm{C}$ for 20 min.

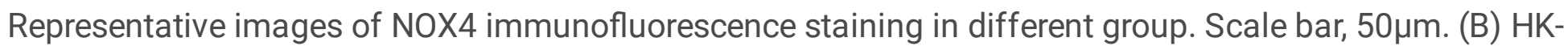
2 cells were pretreated with cytochalasin $\mathrm{D}(1 \mu \mathrm{M})$ followed by 2 hour incubation with AAl-treated, THP-1derived EVa $(1010 / \mathrm{ml})$. Western blot analysis showing the protein expression of NOX4 in different group. 
Dot plots show the corresponding quantification of band intensity and fold change. (C) The protein levels of NOX4 in HK-2 cells stimulated with AAl and treated with human recombinant LRG1(rhLRG1) $(10 \mathrm{ng} / \mathrm{ml})$. Dot plots show the corresponding quantification of band intensity and fold change. (D) Representative images of NOX4 immunofluorescence staining in HK-2 cells stimulated with AAI and

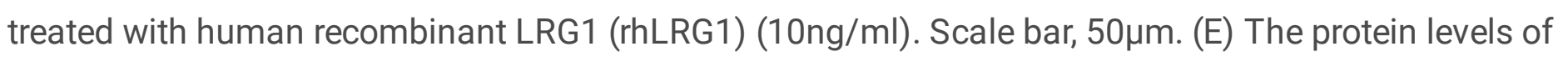
NOX4 in LRG1-pretreated, AAl-induced HK-2 cells treated with siRNA-TGFBR1. Dot plots show the corresponding quantification of band intensity and fold change. (F) Representative images of NOX4 immunofluorescence staining in LRG1-pretreated, AAl-induced HK-2 cells treated with siRNA-TGFBR1. Scale bar, 50 $\mathrm{m}$. Similar results were obtained in triplicate culture assays. $* * P<0.01, * P<0.05$.

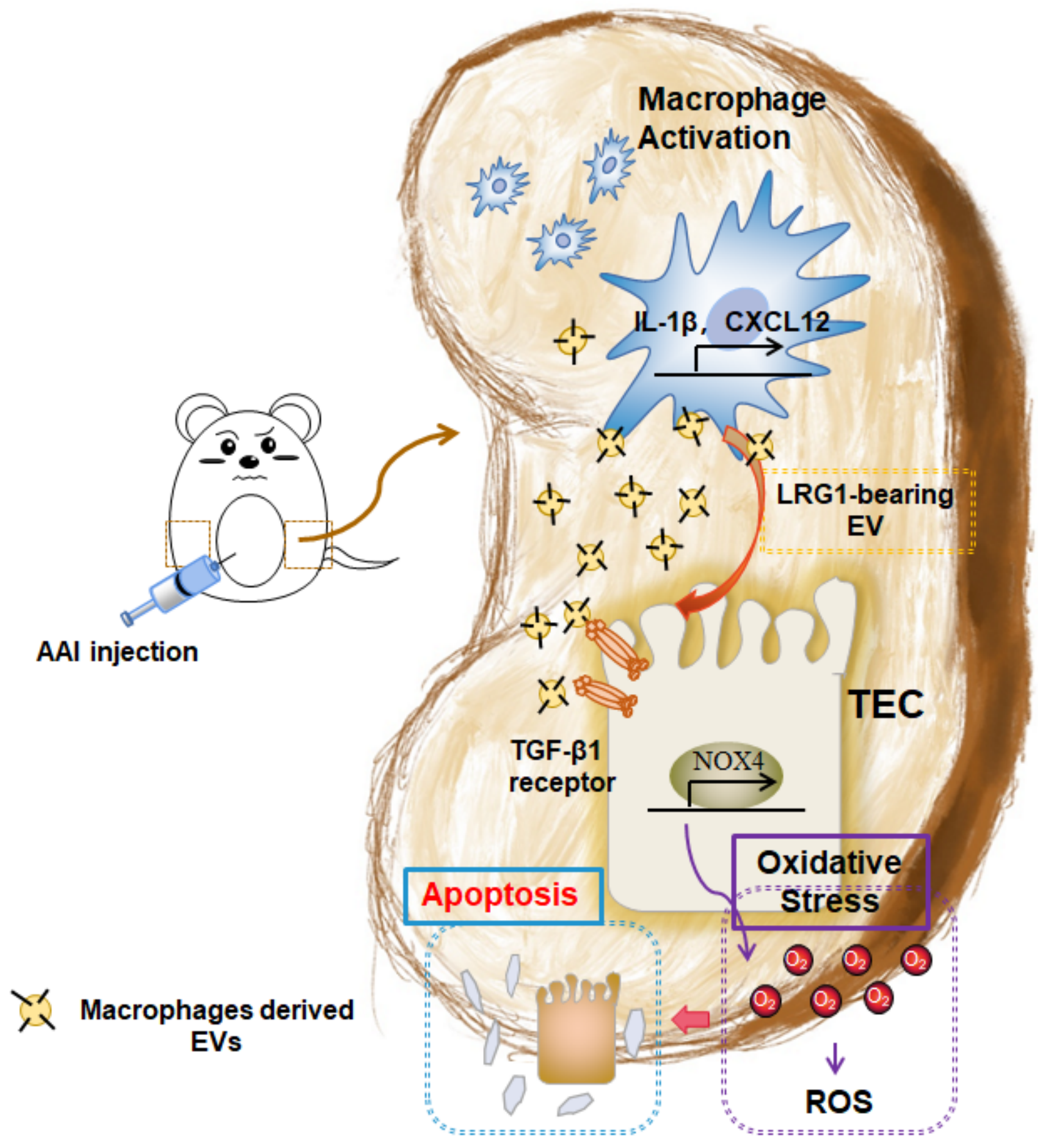

Figure 10 
Macrophages-derived, LRG1-enriched Extracellular Vesicles exacerbate aristolochic acid nephropathy via a TGFßR1-dependent manner by upregulating NOX4

\section{Supplementary Files}

This is a list of supplementary files associated with this preprint. Click to download.

- CBTgraficabstract.pptx

- CBTSupplementalFigures0613.pptx

- CBTSupplementalTables.docx 